EPJ manuscript No.

(will be inserted by the editor)

\title{
The canonical equilibrium of constrained molecular models
}

\author{
Pablo Echenique ${ }^{1,2,3, a}$, Claudio N. Cavasotto ${ }^{4,5}$, and Pablo García-Risueño ${ }^{1,2,3}$ \\ 1 Instituto de Química Física "Rocasolano", CSIC, Serrano 119, E-28006 Madrid, Spain \\ 2 Instituto de Biocomputación y Física de Sistemas Complejos (BIFI), Universidad de \\ Zaragoza, Mariano Esquillor s/n, Edificio I+D, E-50018 Zaragoza, Spain \\ 3 Departamento de Física Teórica, Universidad de Zaragoza, Pedro Cerbuna 12, E-50009 \\ Zaragoza, Spain \\ 4 School of Biomedical Informatics, University of Texas Health Science Center at Houston, \\ 7000 Fannin, Ste. 690, Houston, TX 77030, USA \\ 5 Department of NanoMedicine and Biomedical Engineering, University of Texas Health
} Science Center at Houston, 7000 Fannin, Ste. 690, Houston, TX 77030, USA

\begin{abstract}
In order to increase the efficiency of the computer simulation of biological molecules, it is very common to impose holonomic constraints on the fastest degrees of freedom; normally bond lengths, but also possibly bond angles. Since the maximum time step required for the stability of the dynamics is proportional to the shortest period associated with the motions of the system, constraining the fastest vibrations allows to increase it and, assuming that the added numerical cost is not too high, also increase the overall efficiency of the simulation. However, as any other element that affects the physical model, the imposition of constraints must be assessed from the point of view of accuracy: both the dynamics and the equilibrium statistical mechanics are model-dependent, and they will be changed if constraints are used. In this review, we investigate the accuracy of constrained models at the level of the equilibrium statistical mechanics distributions produced by the different dynamics. We carefully derive the canonical equilibrium distributions of both the constrained and unconstrained dynamics, comparing the two of them by means of a "stiff" approximation to the latter. We do so both in the case of flexible and hard constraints, i.e., when the value of the constrained coordinates depends on the conformation and when it is a constant number. We obtain the different correcting terms associated with the kinetic energy mass-metric tensor determinants, but also with the details of the potential energy in the vicinity of the constrained subspace (encoded in its first and second derivatives). This allows us to directly compare, at the conformational level, how the imposition of constraints changes the thermal equilibrium of molecular systems with respect to the unconstrained case. We also provide an extensive review of the relevant literature, and we show that all models previously reported can be considered special cases of the most general treatments presented in this work. Finally, we numerically analyze a simple methanol molecule in order to illustrate the theoretical concepts in a practical case.
\end{abstract}




\section{Introduction}

Since its first practical applications in the 1950's (see 1] and references therein for a detailed historical account), molecular dynamics (MD) has become a powerful and well-established tool for the study of a wide range of systems, including but not limited to condensed-matter materials [2, fluids 34, polymers and biological molecules [5].

Among the latter, one of the most important families of systems is that of proteins. Together with nucleic acids, proteins are one of the main responsible of the enormous complexity and versatility that living beings exhibit. Although they are mostly constructed as linear chains of only twenty different monomers (the twenty proteinogenic amino acids), and these monomers, in turn, are made of only five types of atoms ( H, C, N, O and S), the finely tuned combination of these elements is capable of producing remarkable 'nano-machines' that perform almost every task that is complex in biological organisms [6. Thus, it is not surprising that a great effort has been invested in the last years in devising new theoretical and computational methods to describe and simulate these important biomolecules. In particular, many groups are pushing forward very ambitious scientific and technological agendas to be able to perform longer and more accurate MD simulations of increasingly larger proteins by different approaches 7889 . In this work, we have in mind the long-term goal of producing more efficient methods for simulating proteins, however, the formalism we introduce and the calculations we perform are directly applicable to any molecular system, as long as it size makes it manageable in present day computers.

The efficiency of a MD simulation is customarily defined as a suitable relation (e.g., the quotient) between its accuracy and its computational cost [10. The overall accuracy can be more finely divided into that related to the physical model used to describe the system and the accuracy of the method used for implementing the dynamics. The computational cost, on the other hand, can be split into that associated with increasing the size of the system and the cost related to the time-propagation of the dynamics [11. The fact that all these issues are intricately coupled is easily seen if we consider examples such as the comparison between ab initio MD based on the ground-state Born-Oppenheimer approximation [1|12 13 14 15] and MD simulations using as energy functions the ones known as classical force fields 16 16 17 18 19:20|21. On the one hand, it is clear that the accuracy of the two physical models is not the same; on the other hand, the size of the systems that can be practically tackled is also different, since quantum chemical methods present a cost that scales typically faster with the number of atoms than that of force fields 2223 . Another example of this interplay between accuracy and cost is the use of coarse-grained descriptions, which, by selecting as interaction centers larger entities than individual atoms, and thus changing the physical model, allow to reach larger systems and longer time-scales than atomistic simulations using either force fields or ab initio methods [24/25/26/27/28].

Yet another technique which can also be used to try to reduce the computational cost of MD simulations by modifying the physical model, and which is the object of this work (and of this special issue of The European Physical Journal), is the imposition of constraints on some of the degrees of freedom of the system. The concept of constraints itself is very general, and it can be used in many ways in the context of molecular modeling and simulation: For example, in Car-Parrinello molecular dynamics [29], which is a type of ab initio MD devised to mimic ground-state BornOppenheimer MD, the time-dependent Khon-Sham orbitals need to be orthonormal; a requirement that can be fulfilled imposing constraints on their scalar product [30. Also, we can use constraints to fix some slow, representative degrees of freedom of our molecular system, also called reaction coordinates, in order to produce free energy

\footnotetext{
a echenique.p@gmail.com
} 
profiles along them that would have taken an unfeasibly long time to be calculated should we have used an unconstrained simulation (see the Blue Moon Ensemble technique in ref. 31 and also a number of related works in this special issue 32 33). In this review, however, we are only concerned with the use of constraints in order to fix the fastest, hardest degrees of freedom of molecular systems (like, e.g., bond lengths or bond angles), with the objective of both reducing the effective size of the conformational space, and allowing for larger time-steps in MD simulations.

The first objective is worth pursuing if we acknowledge that one of the characteristics of large, flexible molecules like proteins (and many others) is that they have an astronomically large conformational space; an issue that may hinder both our understanding of the problem and the possibility of designing algorithms that could efficiently sample this space 343536 . These systems additionally exhibit movements with typical times that span a very wide time-scale, ranging from very fast bond vibrations in the range of tenths of femtoseconds, to the more interesting conformational changes related to biological function (like allosteric transitions or protein folding) which can take times of the order of the millisecond, or even the second [37 38 39]. Since the time-step in MD simulations has to be chosen at least one order of magnitude smaller than the smallest typical time associated to the motion of the system if we want the results to be accurate 3940414243 , this means that we need to calculate $10^{13}-10^{15} \mathrm{MD}$ steps if we want to capture the mentioned biological phenomena in our simulation.

The imposition of constraints that fix the fastest degrees of freedom holds the promise of alleviating both these two problems, however, as any modification of the physical model that describes our system, it may also carry with it a loss of accuracy that renders the simulation useless. In this work, we introduce the rigid and stiff constrained models in order to be able to quantify this loss of accuracy at the level of equilibrium statistical mechanics. In doing that, we benefit from the occasion to thoroughly review the relevant literature, and to try to unify the different, sometimes conflicting, vocabulary and mathematical definitions used to get a handle on the physical concepts. This endeavour is facilitated by the fact that the formalism used in this review is, as far as we are aware, the most general one in the literature, therefore allowing to obtain all previously discussed models as particular cases in which the (often implicit) approximations can be clearly identified.

In sec. 2, we introduce the theoretical framework, beginning by the notation in sec. 2.1. and the Hamiltonian dynamics and statistical mechanics of unconstrained systems in sec. 2.2. Then, we describe and physically justify in sec. 2.3 what types of constraints can be applied, both in terms of the set of coordinates chosen to be constrained (sec. 2.3.1), and in terms of whether or not their equilibrium values are considered to depend on the conformation of the molecule (sec. 2.3.2); we term the constraints flexible if this dependence exists, and hard if the constrained coordinates are assumed to take constant values. In sec. 2.4, we introduce the four possible combinations of constrained statistical mechanics models used in this work and in the literature: the stiff and the rigid model, with either flexible or hard constraints. In sec. 2.5, we comment on the mechanism for comparing the different models and introduce the so-called Fixman potential to do so; also, we collect the different approximations made in the literature with respect to the most general cases discussed in this work, and we establish the relevant relationships among them. In sec. 3, we provide a numerical example in the simple methanol molecule in order to illustrate the concepts, and we benefit from the occasion to also review the previous numerical analyses in the literature. Finally, in sec. 4, we outline the main conclusions of the work, and we mention possible lines of future research. 


\section{Theoretical framework and relation to previous works}

In this section, our objective, which is in fact one of the main objectives of this review, is to lay down the needed mathematical formalism to analyze the equilibrium statistical mechanics of constrained molecular models, and to do so in the most general terms possible.

Of course, we are not the first ones discussing constraints in molecular modeling. As with any other scientific topic, there is a significant degree of arbitrariness in any attempt to fix its historical beginning, but we can talk about a discussion that has been going on at least for three or four decades at the moment of the writing of this manuscript.

Therefore, what is the interest of a new account?

We are confident to be able to convince the reader in the next subsections that, despite the many great works that have dealt with the problem so far, no article defines the problem with the same generality that we display here. This general formalism, which contains all previous descriptions as particular cases, allows us to clearly identify the different approximations that have been assumed in the literature, to relate the many models with one another, and to propose a consistent wording and notation which could facilitate the clarity of future developments.

As we move along, we will indicate at each step which other choices have been made in previous works and how they relate to the general setting.

\subsection{Notation}

The system of interest (which we could call the molecule) is a set of $n$ mass points termed atoms. The Euclidean coordinates of atom $\alpha$ in a frame of reference fixed in the laboratory are denoted by $\mathbf{r}_{\alpha}$ (Vectors of 3 components in the Euclidean 3-dimensional space are denoted by bold symbols.), and its mass by $m_{\alpha}$, with $\alpha=1, \ldots, n$. However, when no explicit mention to the atom index needs to be made, we will use $r^{T}:=$ $\left(r^{\mu}\right)_{\mu=1}^{N}$ to denote the (row) $N$-tuple of all the $N:=3 n$ Euclidean coordinates of the system. By $r$, we shall mean the corresponding column $N$-tuple, or just the ordered set $\left(r^{\mu}\right)_{\mu=1}^{N}$ when matrix operations are not involved. These coordinates parameterize the whole space, denoted by $\mathcal{W}$, and the masses $N$-tuple, $m^{T}:=\left(m_{\mu}\right)_{\mu=1}^{N}$, in such a case, is formed by consecutive groups of three identical masses, corresponding to each of the atom: 1

Apart from the Euclidean coordinates, we shall also use a given set of curvilinear coordinates (also called sometimes general or generalized), denoted by $q^{T}:=\left(q^{\mu}\right)_{\mu=1}^{N}$, to describe the system. The transformation between the two sets and its inverse are respectively denoted by

$$
\begin{aligned}
r^{\mu} & =R^{\mu}(q), \quad \mu=1, \ldots, N, \\
q^{\mu} & =Q^{\mu}(r), \quad \mu=1, \ldots, N,
\end{aligned}
$$

1 Some of the quantities appearing in the formalism, such as the velocities $\dot{r}^{\mu}$, the displacements $\delta r^{\mu}$, or the mass-metric tensor $G_{\mu \nu}$ (see the following sections), change like tensors of some type under a general change of coordinates; in such cases, the position of the indices is dictated by the tensor type (1-time contravariant tensors in the case of $\dot{r}^{\mu}$ or $\delta r^{\mu}, 2$-times covariant in the case of $G_{\mu \nu}$ ). Some other quantities, such as $\ddot{r}^{\mu}, F_{\mu}, m_{\mu}$ or $r^{\mu}$, do not change like any type of tensorial object under a general change of coordinates; in such cases, the position of the indices is chosen according to notational convenience, or to the way in which they transform under some particular family of changes (e.g., linear ones). 
and we will assume that, for the points of interest, this is a proper change of coordinates, i.e., that the Jacobian matrix

$$
J_{\nu}^{\mu}:=\frac{\partial R^{\mu}(q)}{\partial q^{\nu}}
$$

has non-zero determinant, being its inverse

$$
\left(J^{-1}\right)_{\nu}^{\mu}=\frac{\partial Q^{\mu}(r)}{\partial r^{\nu}}
$$

Although the curvilinear coordinates $q$ are a priori general, it is very common to take profit from the fact that the typical potential energy functions of molecular systems in absence of external fields do not depend on the overall position or orientation, i.e., they are invariant under overall translations and rotations, in order to choose a set of curvilinear coordinates split into $q^{T}=\left(e^{T}, w^{T}\right)$, where the first six, $e^{T}:=\left(e^{A}\right)_{A=1}^{6}$ are any external coordinates that parameterize the aforementioned global position and orientation of the system ${ }^{2}$. The remaining $N-6$ coordinates $w^{T}:=\left(w^{a}\right)_{a=7}^{N}$ are called internal coordinates and determine the positions of the atoms in some frame of reference fixed in the system. They parameterize what we shall call the internal subspace, denoted by $\mathcal{I}$, and the coordinates $e$ parameterize the external subspace, denoted by $\mathcal{E}$; hence, we can split the whole space as $\mathcal{W}=\mathcal{E} \times \mathcal{I}$ (denoting by $\times$ the Cartesian product of sets).

The reader is probably familiar with the use of frames of reference fixed in the system based on the center of mass and the principal axes of inertia, but, although this is well adapted to the parameterization of the dynamics of rigid bodies [4], it is less so to flexible entities like molecular systems, where the relative distances of their constituents are not constant in time. Following ref. 45, we define a more suitable frame of reference fixed in the system to perform some of the calculations, although it is worth stressing that most of the mathematical formalism introduced in this work should not depend on this choice as long as the external and internal subspaces are well separated.

To define this frame of reference, we select three atoms (denoted by 1, 2 and 3 in Fig. 1) in such a way that $\mathbf{o}$, the position in the laboratory frame of reference of the origin of the frame of reference fixed in the system, is the Euclidean position of atom 1 (i.e., $\mathbf{o}:=\mathbf{r}_{1}$ ). The orientation of the frame of reference $\left(x^{\prime}, y^{\prime}, z^{\prime}\right)$ fixed in the system is chosen such that atom 2 lies in the positive half of the $z^{\prime}$-axis, and atom 3 is contained in the $\left(x^{\prime}, z^{\prime}\right)$-plane, with projection on the positive half of the $x^{\prime}$-axis (see Fig. 1). The position of any given atom $\alpha$ in the new frame of reference fixed in the system is denoted by $\mathbf{r}_{\alpha}^{\prime}$, and we have that the previously unspecified external coordinates are now $e^{T}:=\left(e^{A}\right)_{A=1}^{6}=\left(o_{x}, o_{y}, o_{z}, \phi, \theta, \psi\right)$, where $(\phi, \theta, \psi)$ are three 'Euler angles' that parameterize the orientation of the 'primed' axes with respect to the 'unprimed' ones.

More explicitly, if $E(\phi, \theta, \psi)$ is the Euler rotation matrix (in the ZYZ convention) that takes a free 3 -vector of primed components, $\mathbf{a}^{\prime}$, to the frame of reference fixed in the laboratory, i.e., $\mathbf{a}=E(\phi, \theta, \psi) \mathbf{a}^{\prime}$, its explicit expression is the following [44:

$$
E(\phi, \theta, \psi)=\underbrace{\left(\begin{array}{ccc}
\cos \phi & -\sin \phi & 0 \\
\sin \phi & \cos \phi & 0 \\
0 & 0 & 1
\end{array}\right)}_{\Phi(\phi)} \underbrace{\left(\begin{array}{ccc}
-\cos \theta & 0 & \sin \theta \\
0 & 1 & 0 \\
-\sin \theta & 0 & -\cos \theta
\end{array}\right)}_{\Theta(\theta)} \underbrace{\left(\begin{array}{ccc}
\cos \psi-\sin \psi & 0 \\
\sin \psi & \cos \psi & 0 \\
0 & 0 & 1
\end{array}\right)}_{\Psi(\psi)} .
$$

2 The practice adopted in this section of using different groups of indices, as well as different symbols, for the individual coordinates in each one of the sets $r, q, e$, etc., allows us to grasp at a first glimpse the range of values in which each of the indices vary. See table 1 for a summary of the indices used, the symbols denoting the sets of coordinates, their meaning and the spaces parameterized by them. 


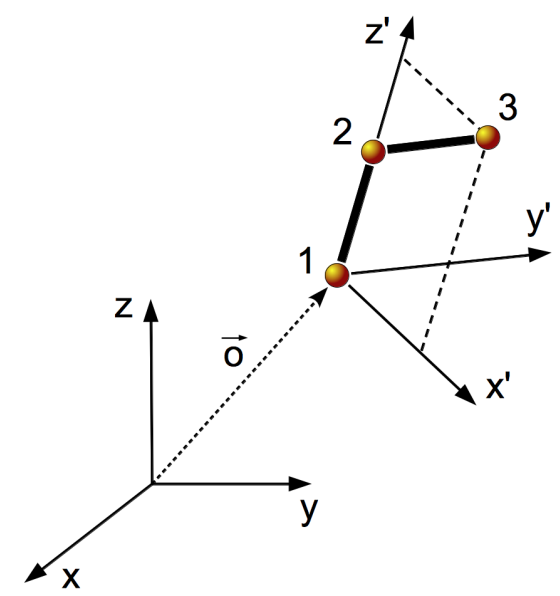

Fig. 1. Definition of the frame of reference fixed in the system.

The position, $\mathbf{r}_{\alpha}^{\prime}$, of any given atom $\alpha$ in the axes fixed in the system is by construction a function, $\mathbf{R}_{\alpha}^{\prime}(w)$, of only the internal coordinates $w$, and the transformation from the Euclidean coordinates $r$ to the curvilinear coordinates $q$ in (1a) may be written as follows:

$$
\mathbf{r}_{\alpha}=\mathbf{R}_{\alpha}(q)=\mathbf{o}+E(\phi, \theta, \psi) \mathbf{R}_{\alpha}^{\prime}(w) .
$$

An additional definition of subsets of the coordinates $q$ is motivated by the imposition of constraints. Assume that we impose $L=N-K$ independent, holonomic and scleronomous constraints to the system:

$$
\sigma^{I}(q)=0, \quad I=K+1, \ldots, N .
$$

The usual definition [44] is that the constraints are called holonomic if they can be written in the form $\sigma^{I}(q, t)=0$, and scleronomous when the functions $\sigma^{T}(q):=\left(\sigma^{I}(q)\right)_{I=K+1}^{N}$ do not depend explicitly on time $t$. They are independent if their gradients $\left(\left(\partial \sigma^{I} / \partial q^{\mu}\right)(q)\right)_{\mu=1}^{N}, I=K+1, \ldots, N$, constitute $L$ linearly independent vectors of $N$ components for every point $q$ satisfying the constraints [i.e., for every point $q$ such that $\left.\sigma^{I}(q)=0, I=K+1, \ldots, N\right]$; or, otherwise stated, the constraints are independent if the matrix with entries $\left(\partial \sigma^{I} / \partial q^{\mu}\right)(q)$ has range $L$. In such a case, the constraints uniquely define (at least locally) a subspace of $\mathcal{W}$ of constant dimension $N-L=K$, called the constrained subspace and denoted by $\mathcal{K}$.

Moreover, this independence condition allows, in the vicinity of each point $q$ and by virtue of the Implicit Function Theorem [46 47, to (formally) solve eq. (6) for $L$ of the coordinates $q$, which we arbitrarily place at the end of $q$, splitting the original set as $q^{T}=\left(u^{T}, d^{T}\right)$, with $u^{T}:=\left(u^{r}\right)_{r=1}^{K}$ and $d^{T}:=\left(d^{I}\right)_{I=K+1}^{N}$. Then, in the vicinity of each point $q$ satisfying (6), we can express the relations defining the constrained subspace, $\mathcal{K}$, parametrically by

$$
d^{I}=f^{I}(u), \quad I=K+1, \ldots, N
$$

where the functions $f^{T}(u):=\left(f^{I}(u)\right)_{I=K+1}^{N}$ are the ones whose existence the Implicit Function Theorem guarantees. The coordinates $u$ are thus termed unconstrained and they parameterize $\mathcal{K}$, whereas the coordinates $d$ are called constrained and their value is determined at each point of $\mathcal{K}$ according to (7). As we will see later, in the most 
Table 1. Symbols and indices used for the different sets of coordinates, as well as the names of the spaces parameterized by them.

\begin{tabular}{llllll} 
Symbol & Indices & Range & Number & Name & Space \\
\hline $\mathbf{r}$ & $\alpha, \beta, \gamma, \ldots$ & $1, \ldots, n$ & $n$ & Atoms & - \\
$r, q$ & $\mu, \nu, \rho, \ldots$ & $1, \ldots, N$ & $N=3 n$ & Whole space & $\mathcal{W}$ \\
$e$ & $A, B, C, \ldots$ & $1, \ldots, 6$ & 6 & External & $\mathcal{E}$ \\
$w$ & $a, b, c, \ldots$ & $6+1, \ldots, N$ & $N-6$ & Internal & $\mathcal{I}$ \\
$s$ & $i, j, k, \ldots$ & $6+1, \ldots, K$ & $M$ & Unconstrained internal & $\Sigma$ \\
$d$ & $I, J, K, \ldots$ & $K+1, \ldots, N$ & $L=N-K$ & Constrained internal & - \\
$u$ & $r, s, t, \ldots$ & $1, \ldots, K$ & $K$ & Unconstrained & $\mathcal{K}$
\end{tabular}

general case, the functions $f$ will depend on $u$, and the constraints will be said to be flexible. In the particular case in which all the functions $f$ are constant along $\mathcal{K}$, the constraints are called hard, and all the calculations are considerably simplified.

Of course, even if $\mathcal{K}$ is regular in all of its points, the particular coordinates $d$ that can be solved need not to be the same along the whole space ${ }^{3}$. Nevertheless, we will assume this to be the case throughout this work, as it is normally implicitly done in the literature [48950, and thus we will consider that $\mathcal{K}$ is parameterized by the same subset of unconstrained coordinates $u$ in all of its points 4 .

Although in general the functions $\sigma(q)$ in (6) may involve all the coordinates $q$, the already mentioned property of invariance of the potential energy function [or the potential energy plus a term related to the determinant of the whole-space mass-metric tensor (see sec. 2.4.3)] under changes of the external coordinates, $e$, together with the fact (which we shall discuss later) that the potential energy (or the aforementioned object) can be regarded as 'producing' the constraints, make physically frequent the definition of constraints affecting only the internal coordinates, $w$ :

$$
\sigma^{I}(w)=0
$$

In such a situation, which will be the one treated in this work, the constrained coordinates, $d$, are contained into the internal ones, $w$, so that the latter can be split as $w^{T}=\left(s^{T}, d^{T}\right)$, where the first $M:=K-6=N-L-6$ ones, $s^{T}:=\left(s^{i}\right)_{I=6+1}^{K}$, are called constrained internal coordinates and parameterize the constrained internal subspace, denoted by $\Sigma$. Hence, the constrained subspace can be split as $\mathcal{K}=\mathcal{E} \times \Sigma$, and the analogue to (7) can be written for this case as

$$
d^{I}=f^{I}(s)
$$

Finally, if the constraints in (9) are used, together with (5), the Euclidean position of any atom may be parameterized with the set of all unconstrained coordinates, $u$,

3 One of the simplest examples of this being the circle in $\mathbb{R}^{2}$, which is given by $f(x, y):=$ $x^{2}+y^{2}-R^{2}=0$, an implicit expression whose gradient is non-zero for all $(x, y) \in \mathcal{K}$. However, if we try to solve, say, for $y$ in the whole space $\mathcal{K}$, we will run into trouble at $y=0$; if we try to solve for $x$, we will find it to be impossible at $x=0$. I.e., the Implicit Function Theorem does guarantee that we can solve for some of the original coordinates at each regular point of $\mathcal{K}$, but sometimes the solved coordinate has to be $x$ and sometimes it has to be $y$.

4 Note that this qualification is unnecessary in the hard case introduced in sec. 2.4.2. where the uncoupled nature of the functions $\sigma(q)$ makes it possible to solve for the same coordinates at every point. 
as follows:

$$
\begin{aligned}
\mathbf{r}_{\alpha} & =\boldsymbol{\mathcal { R }}_{\alpha}(u):=\mathbf{R}_{\alpha}(e, s, f(s))=\mathbf{o}+E(\phi, \theta, \psi) \mathbf{R}_{\alpha}^{\prime}(s, f(s))= \\
& =: \mathbf{o}+E(\phi, \theta, \psi) \boldsymbol{\mathcal { R }}_{\alpha}^{\prime}(s),
\end{aligned}
$$

where the name of the transformation functions has been changed from $R$ to $\mathcal{R}$, and from $R^{\prime}$ to $\mathcal{R}^{\prime}$, in order to emphasize that the dependence on the coordinates is different between the two cases.

\subsection{Hamiltonian dynamics and statistical mechanics without constraints}

Let us now briefly introduce the formalism needed to tackle the dynamics and statistical mechanics of any molecular system such as the one described in the previous section. We do this only to fix the notation and to write some expressions that will be used later; the interested reader might want to check any of the classical texts in the subject, such as [5144152].

The central object that determines the behaviour of a classical system is the Hamiltonian function. If one starts by writing the Lagrangian function in terms of the Euclidean coordinates $r$ and velocities $\dot{r}$ (with the over-dot denoting the time derivative),

$$
L_{\mathrm{Euc}}(r, \dot{r}):=\frac{1}{2} m_{\mu}\left(\dot{r}^{\mu}\right)^{2}-U(r),
$$

where $U(r)$ is the potential energy of the system and the convention prescribing summation on repeated indices has been used, then the Euclidean Hamiltonian can be easily obtained through the usual Legendre transform:

$$
H_{\mathrm{Euc}}(r, \pi):=\frac{1}{2 m_{\mu}}\left(\pi_{\mu}\right)^{2}+U(r),
$$

where $\pi$ are the Euclidean momenta defined as

$$
\pi_{\mu}:=\frac{\partial L_{\mathrm{Euc}}}{\partial \dot{r}^{\mu}}=m_{(\mu)} \dot{r}^{(\mu)}, \quad \mu=1, \ldots, N,
$$

with the parentheses around the indices indicating that the convention that prescribes summation when indices are repeated is not to be followed.

The dynamics of the system, once the Hamiltonian in eq. 12 is known, is given by the solutions of a set of coupled, first-order differential equations known as Hamilton's equations:

$$
\begin{aligned}
& \dot{r}^{\mu}=\frac{\partial H_{\mathrm{Euc}}(r, \pi)}{\partial \pi_{\mu}}, \\
& \dot{\pi}_{\mu}=-\frac{\partial H_{\mathrm{Euc}}(r, \pi)}{\partial r^{\mu}} .
\end{aligned}
$$

If we now want to describe this dynamics in terms of the curvilinear coordinates $q$ introduced in sec. 2.1. which are better adapted to the covalent connectivity of molecular systems, we can start by taking the time derivative of the change of coordinates in eq. 1aa),

$$
\dot{r}^{\mu}=\frac{\partial R^{\mu}(q)}{\partial q^{\nu}} \dot{q}^{\nu}
$$


and also noticing that, for any given potential energy $U(r)$ expressed as a function of $r$, we can perform the same change of coordinates and define the potential energy as a function of $q$ by

$$
V(q):=U(R(q)),
$$

which, as we mentioned, typically only depends on the internal ones, i.e., $V(q)=$ $V(w)$.

If we take these last two expressions to eq. (11), we arrive to the Lagrangian in curvilinear coordinates, or unconstrained Lagrangian, as we will call it in the rest of the manuscript:

$$
L(q, \dot{q}):=\frac{1}{2} \dot{q}^{\nu} G_{\nu \rho}(q) \dot{q}^{\rho}-V(w),
$$

where the whole-space mass-metric tensor $(\mathrm{MMT}) G_{\nu \rho}(q)$ is defined as

$$
G_{\nu \rho}(q):=\frac{\partial R^{\mu}(q)}{\partial q^{\nu}} m_{\mu} \frac{\partial R^{\mu}(q)}{\partial q^{\rho}} .
$$

Following again the usual process of the Legendre transform, we can derive from eq. (17) the unconstrained Hamiltonian,

$$
H(q, p):=\frac{1}{2} p_{\nu} G^{\nu \rho}(q) p_{\rho}+V(w),
$$

where $G^{\nu \rho}(q)$ is the inverse of the whole-space MMT in eq. (18) and the canonical conjugate momenta are defined as

$$
p_{\mu}:=\frac{\partial L}{\partial \dot{q}^{\mu}}=G_{\mu \nu}(q) \dot{q}^{\nu} .
$$

Finally, the Hamilton equations in terms of the curvilinear coordinates and momenta are formally identical to those in eqs. (14):

$$
\begin{aligned}
& \dot{q}^{\mu}=\frac{\partial H(q, p)}{\partial p_{\mu}}, \\
& \dot{p}_{\mu}=-\frac{\partial H(q, p)}{\partial q^{\mu}},
\end{aligned}
$$

Under the usual assumptions of ergodicity and equal a priori probabilities [52, it can be shown that the partition function characterizing the statistical mechanics equilibrium of such a dynamics in the canonical ensemble (i.e., at constant volume $V$, number of particles $n$, and temperature $T$ ) is given by

$$
Z=\frac{\alpha_{\mathrm{QM}}}{h^{N}} \int e^{-\beta H(q, p)} \mathrm{d} q \mathrm{~d} p,
$$

where $h$ is Planck's constant, we denote $\beta:=1 / R T$ (per mole energy units are used throughout the article, so $R T$ is preferred over $k_{B} T$ ) and $\alpha_{\mathrm{QM}}$ is a combinatorial number that accounts for quantum indistinguishability and that must be specified in each particular case (e.g., for a gas of $n$ indistinguishable particles, $\alpha_{\mathrm{QM}}=1 / n$ !). With $\int \mathrm{d} q \mathrm{~d} p$, we denote integration over all positions $q^{\mu}$ and their respective momenta $p_{\mu}$, for $\mu=1, \ldots, N$, being the range of integration usually from $-\infty$ to $\infty$ for the momenta, and the appropriate one for each position. For example, if a typical scheme for defining the internal coordinates, such as the SASMIC one [45, is used, bond lengths must be integrated from 0 to $\infty$, bond angles from 0 to $\pi$, and dihedral angles from $-\pi$ to $\pi$. 
The corresponding equilibrium probability density function (PDF) is thus given by

$$
P(q, p)=\frac{e^{-\beta H(q, p)}}{\int e^{-\beta H\left(q^{\prime}, p^{\prime}\right)} \mathrm{d} q^{\prime} \mathrm{d} p^{\prime}},
$$

being $P(q, p) \Delta q \Delta p$ interpreted as the probability of finding the system with positions in $(q, q+\Delta q)$, and momenta in $(p, p+\Delta p)$, for sufficiently small $\Delta q, \Delta p$.

In the same sense, the equilibrium average of any observable $O(q, p)$ is

$$
\langle O\rangle=\int O(q, p) P(q, p) \mathrm{d} q \mathrm{~d} p
$$

It is also common in the literature to study observables, or properties, which depend only on the positions $q$ and not on the momenta $p$; the native conformation of a protein being a notable example of this 6 . In such a case, we can appeal to the well-known formula for the $N$-dimensional Gaussian integral [53],

$$
\int_{-\infty}^{\infty} \cdots \int_{-\infty}^{\infty} e^{-\frac{1}{2} x^{\mu} M_{\mu \nu} x^{\nu}+v_{\mu} x^{\mu}} \mathrm{d} x=\sqrt{\frac{(2 \pi)^{N}}{\operatorname{det} M}} e^{\frac{1}{2} v_{\mu} M^{\mu \nu} v_{\nu}}
$$

where $M$ is an $N \times N$ matrix (that must be positive definite in order for the integral to be finite), $M^{\mu \nu}$ denotes the entries of $M^{-1}$, and $v$ is a (possibly null) $N$-tuple, to 'integrate out' the momenta in the partition function in eq. (22), yielding:

$$
Z=\chi(T) \int e^{-\beta\left[V(w)-T \frac{R}{2} \ln \operatorname{det} G(q)\right]} \mathrm{d} q,
$$

where

$$
\chi(T):=\left(\frac{2 \pi}{\beta}\right)^{N / 2} \frac{\alpha_{\mathrm{QM}}}{h^{N}} .
$$

If we perform the same integration in the joint PDF in eq. (23), the factor $\chi(T)$ cancels out and we arrive to the marginal equilibrium PDF in the space of the positions $q$ :

$$
P(q)=\frac{e^{-\beta\left[V(w)-T \frac{R}{2} \ln \operatorname{det} G(q)\right]}}{\int e^{-\beta\left[V\left(w^{\prime}\right)-T \frac{R}{2} \ln \operatorname{det} G\left(q^{\prime}\right)\right]} \mathrm{d} q^{\prime}}=: \frac{e^{-\beta F(q)}}{\int e^{-\beta F\left(q^{\prime}\right)} \mathrm{d} q^{\prime}},
$$

where the normal abuse of notation in probability theory has been committed, using the same symbol, $P$, for the two different functions in eqs. 23) and 28, and where we have defined

$$
\begin{aligned}
F(q) & :=V(w)-T S^{\mathrm{k}}(q), \\
S^{\mathrm{k}}(q) & :=\frac{R}{2} \ln \operatorname{det} G(q) .
\end{aligned}
$$

The notation in the equations above is intentional, in the sense that $F(q)$ can be interpreted as a free or effective energy, since it is obtained via the elimination of some degrees of freedom (the momenta $p$ ), and it therefore describes the energetics (at least the equilibrium one) of the remaining degrees of freedom (the positions $q$ ) in a sort of mean-field of the ones that have been eliminated. This free or effective character is also emphasized by the fact that $F(q)$ depends on the temperature $T$, even if in a simple way, and the analogy can be taken one step further if we regard $V(q)$ as an internal energy and the correcting term as a kinetic entropy [54; something which is compatible with its being linear in $R T$. 
Again, the meaning of this last marginal PDF in eq. $(28)$ is made explicit if we note that $P(q) \Delta q$ is the probability of finding the system with positions in $(q, q+\Delta q)$, for sufficiently small $\Delta q$ (irrespective of the value of the momenta), and that the equilibrium average of any momenta-independent observable $O(q)$ is given by

$$
\langle O\rangle=\int O(q) P(q) \mathrm{d} q .
$$

Now, as we mentioned in sec. 2.1. the potential energy of molecular systems in absence of external fields is typically independent of the external coordinates $e$, and this is why we have written $V(w)$ and not $V(q)$ in all the previous expressions. The whole-space MMT $G(q)$ in eq. $\sqrt{18}$ on the other hand, and in particular its determinant in eqs. (26) and (28), does depend on the external coordinates $e$. It is thus convenient, in the case that we are also dealing with observables $O(w)$ which are independent of the external coordinates, to try to eliminate them from the expressions if possible. One can indeed formally always integrate over the external coordinates (or any other variables spanning the probability space) in order to get to the corresponding marginal PDF in the internal space $\mathcal{I}$, i.e., depending only on the internal coordinates $w$. However, until recently, it was not clear if this process could be performed analytically (specially for the more involved, constrained case in sec. 2.4.1), thus yielding manageable final expressions. In a previous work by some of us [55], we settled the issue proving that this is in fact possible and providing the exact analytical expressions to be used for the marginal $\mathrm{PDF}$ in $\mathcal{I}$.

To integrate out the external coordinates analytically from $\operatorname{det} G(q)$, we only need to realize that, since the unconstrained case can be trivially assimilated to a constrained situation with the number of constraints, $L=0$, all the results regarding the factorization of the induced MMT determinant that we will introduce later in sec. 2.4.1 apply, and we have the unconstrained analogue of eq. (64):

$$
\operatorname{det} G(q)=\sin ^{2} \theta \operatorname{det} G^{\prime}(w)
$$

with $G^{\prime}(w)$ the $N \times N$ matrix obtained from eq. 65 if the unconstrained internal coordinates $s$ are substituted by all the internal coordinates $w$, and $\theta$ being one of the Euler angles introduced in sec. 2.1.

Now, the external angle $\theta$ can be integrated both in the numerator and in the denominator of eq. (28), yielding the marginal PDF in the internal space:

$$
P(w)=\frac{e^{-\beta F(w)}}{\int e^{-\beta F\left(w^{\prime}\right)} \mathrm{d} w^{\prime}},
$$

where we have defined, analogously to eq. (29),

$$
\begin{aligned}
F(w) & :=V(w)-T S^{\mathrm{k}}(w), \\
S^{\mathrm{k}}(w) & :=\frac{R}{2} \ln \operatorname{det} G^{\prime}(w) .
\end{aligned}
$$

Finally, let us remark that every step taken in this section, from the original Hamiltonian in eq. (19) to the marginal PDF in eq. $\sqrt{32}$, is exact. The approximations will arrive when we introduce the constrained models in the following sections.

\subsection{Types of constraints}

If we assume that the constraints we shall impose on our physical system can be expressed as in $(9)$, where $w:=(s, d)$ are typical internal coordinates, such as the 
ones defined in ref. 45, then the different types of constraints that can be imposed may be classified according to two independent criteria, which, together, constitute a definition of the internal constrained subspace $\Sigma$, namely, (a) What coordinates are constrained? I.e., what coordinates comprise the set $d$ ? and (b) Do the values of the constrained coordinates in $\Sigma$ depend on the point $s$, or not?

\subsubsection{What coordinates are constrained}

Regarding (a), it is typical in the literature to constrain whole groups of coordinates of the same type, classifying them both according to whether they are bond lengths, bond angles, etc., and also in terms of the atoms involved in their definition. For example, in ref. [8], bond-lengths involving Hydrogen atoms are constrained in a $20 \mu \mathrm{s}$ MD of the Villin Headpiece; in ref. [7, all bond-lengths are constrained to study the folding of a modified version of the same protein; in ref. [56] all bond-lengths as well as bond angles of the form $\mathrm{H}-\mathrm{O}-\mathrm{X}$ or $\mathrm{H}-\mathrm{X}-\mathrm{H}$, being $\mathrm{X}$ any atom, are constrained to simulate an 80-residue protein; the same scheme of constraints is applied in ref. [57]; and we have the constraining of everything but torsion angles in the so-called torsion-angle $M D$ 58 59606162 .

If the system is close to a local minimum of the potential energy $V(w)$, this way of proceeding is based on the intuition that we can associate similar vibrational frequencies to internal coordinates of the same type, with little influence of the local environment surrounding the atoms that define them. In this way, if we start by constraining the highest-frequency coordinates, we remove their vibrations from the dynamics, and, as we mentioned in sec. 1, we can use a larger time-step to integrate the equations of motion. This is only intuitive and not rigorous because (i) the idea is also used away from minima, where 'vibrational frequencies' are not properly defined, (ii) even close to a minimum, the vibrational modes of molecules cannot be directly associated to individual internal coordinates, but to linear combinations of them, and (iii) even if a vibrational mode is almost purely associated to a given internal coordinate, its vibrational frequency does depend on the environment of the atoms defining it; the question is how much.

It is therefore probably a more accurate way of proceeding to define the constrained coordinates in an adaptive manner, depending on the conformation of the system; but such schemes either do not exist or they are not very popular in the literature as far as we are aware. Therefore, although we remark that any choice of constrained coordinates is in principle possible as long as the resulting model is physically accurate, we will be thinking here in the simpler case of constraining whole groups of internal coordinates of the same type (defining type in the common and chemically intuitive way specified above).

Regarding this point, it is also worth remarking that, although we argued in sec. 2.1 that, by virtue of the Implicit Function Theorem, it is locally equivalent to express the constraints implicitly, as in eq. (8), or parametrically, as in eq. (9), the developments that can be performed, the final expressions, and the practical algorithms derived from them are different in the two cases. We will base all the discussion on the formalism in which the coordinates to be constrained, $d$, can be identified and the constraints written as in eq. (9), using the unconstrained coordinates, $u$, to parameterize the constrained subspace. In such a case, it is most natural to assume that these coordinates, $q:=(u, d)$, are the typical ones used in molecular simulation 45, however, the possibility also exists to use the implicit constraints in eq. (8) and work in Euclidean coordinates. In fact this is the version that is needed for the Lagrange multipliers formulation in the whole space and for the implementation of the vast majority of practical algorithms, both in the flexible and hard cases discussed later 
63 49. Related to this, one can also choose a set of modified curvilinear coordinates $\tilde{q}$ in which the constrained ones are given exactly by the $\sigma$ functions in eq. (8), and therefore the constraints are expressed just as $\tilde{d}=\dot{\tilde{d}}=0$. We will make frequent references to these other choices as we advance in the discussion.

\subsubsection{Flexible vs. hard constraints}

Once the constrained coordinates $d$ have been selected, we can ask question (b): Do their values in $\Sigma$ depend on the point or not? I.e., do they depend on the unconstrained internal coordinates $s$ ? We shall call the constraints flexible if the answer is 'yes' and hard if it is 'no'.

It is worth mentioning at this point that the wording used to refer to constrained systems in the literature is multiple and often misleading. One of the aims of this work is to clarify the mathematical definitions behind the words and to provide a consistent vocabulary to refer to the different types of models. What we have called flexible constraints, for example, are also called flexible in refs. [41/64/65/49/50/66], elastic in 67, adiabatic in 68, and soft in 69411507068; whereas the hard case is also called hard in refs. 694164150|68, just constrained in 65, holonomic in 67|68, rigid in [49:50, and fully constrained in [50. In the light of the more formal discussion in what follows, the reader will appreciate that some of these terms are clearly misleading (elastic, holonomic or fully constrained), and, in any case, so many names for such simple concepts is detrimental for the understanding in the field. Even if the reader does not like the choice advocated in this work, she should recognize that a unification of the vocabulary is desirable.

The situation is further complicated by the fact that, when studying the statistical mechanics of constrained systems, as we will also see later in great detail, one can think about two different models for calculating the equilibrium PDF. The names of these models often collide with the ones used for defining the type of constraints applied. On the one hand, one can implement the constraints by the use of very steep potentials around the constrained subspace; a model often called flexible [71/72|73|74/75/76/77] (the worst option due to vocabulary clashes), sometimes called soft [5178, and sometimes stiff [79|80|5|48|81/82/83] (as we advocate in this work). On the other hand, one can assume D'Alembert's principle [4|84] and hypothesize that the forces are just the ones needed for the system to never leave the constrained subspace during its dynamical evolution; a model normally called rigid [71|79|80|72|48|73|74|75|76|85|77|82|83, as we do here, but sometimes also hard [5178|65], which is a bad choice in terms of clashes. This interference between the two families of wording is entirely unjustified, since, as we will see later, the two types of constraints and the two types of statistical mechanics models can be independently combined; one can have either the stiff or the rigid model, with either flexible or hard constraints.

As we briefly mentioned in sec. 2.1. the most general case from the mathematical point of view is indeed that of flexible constraints, where the functions $f$ in $(9)$ are not constant numbers, and it is also the case that arises more naturally from physical considerations.

Indeed, one way of justifying a given constrained model is by comparing it to the original, unconstrained one. This choice of the reference is not the only possible one; it is also legitimate to compare the constrained models introduced in this work directly to experimental results, or to more fundamental theoretical descriptions such as those based in quantum mechanics. However, we (and many others [76|58|85|64|67|59|41|86|87|71|69|88|89|90|77|69|91|50]) have preferred to use the classical unconstrained model as a reference to be able to assess the influence of the imposition of constraints in an incremental way consisting of a series of controlled 
steps. Although some works have advocated the point of view that the proper justification of constrained models should come from a quantum mechanical treatment

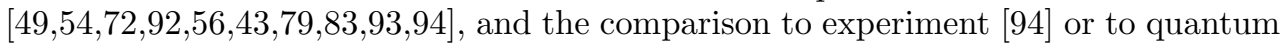
mechanics is indeed more relevant in terms of the absolute accuracy of the constrained models, the approximations connecting the former to the latter are many, thus obscuring the influence of each individual step. In particular, it is worth mentioning refs. 72957379 for a clear exposition of the many arguments that appear when the comparison is of this second, more convoluted class. This is much so that we can find works in the literature that, based on quantum mechanical arguments, favour the classical stiff model (introduced in sec. 2.4.3) against the rigid one (sec. 2.4.1) 72, or vice versa 49. Before these discrepancies can be solved and a reference classical model can be rigorously derived from quantum mechanics (see refs. 9697/9899100101/102/103/73/81 and also the review by Álvarez-Estrada and Calvo in this special issue 104 for the most advanced attempts to do so), we will consider the unconstrained dynamics as a more or less 'safe harbor', and as the reference to which assess the classical constrained models discussed in this work. It is also worth remarking that, in doing so, we are also sidestepping the question about the accuracy of the potential energy itself (since we compare to the unconstrained dynamics given by the same potential). Although this is an important issue affecting the absolute accuracy of the results, and it is very likely that classical force fields are not enough to describe some phenomena, e.g., those involving charge transfer [15], we can consider the two sources of error separately and analyze each one of them at a time.

In this spirit, we may think that the fact that allows us to treat some coordinates as constrained and some others as unconstrained is that the former need more energy to be separated from their minimum-energy values than the latter, and thus this separation is more unlikely to occur. In principle, these minimum-energy values as well as the energy cost needed to separate the system from them can be defined both including the momenta or excluding them, and this can be done either at the dynamical or at the statistical mechanics level.

In this work, we are mainly concerned with the equilibrium properties of constrained models and we point the reader interested in dynamical considerations to 694110567964010673717782107 , as well to refs. 28108109110 in this same special issue. In brief, we want to remark that the approach followed in this work is in general simpler and it is subject to less uncertainties than dynamical analyses, but, on the other hand, its application must be circumscribed to the computation of equilibrium averages.

In equilibrium statistical mechanics, all the information is contained in the different partition functions [such as (22)] and PDFs [such as (23)], and all observable quantities are calculated through integrals, such as (24). Therefore, it is most natural to look for the regions in which the quantity under the integral sign is non-negligible, and define our constrained subspace exactly as those regions. Assuming that the observables $O(q, p), O(q)$ are smooth enough functions of their arguments, the constrained subspace so defined corresponds to the values of the constrained coordinates that maximize the corresponding PDFs for every value of the rest of variables. Once this information is used to define the constrained subspace [i.e., to answer question (b)], the issue about how to sample the conformations of the system to produce the appropriate equilibrium PDF becomes an algorithmic one (we can use Monte Carlo techniques, MD, etc.). If the algorithm used to do the sampling is, as it is often the case, a dynamical one, then nothing in the discussed approach guarantees that the trajectories obtained are similar in any way to those of the unconstrained system. The only thing that we can be sure of is that the equilibrium averages will be in- 
deed consistent with the approximations involved in the definition of the constrained subspace as that of maximum probability.

In this framework, we have several options to define the most probable values of the constrained coordinates $d$, i.e., the functions $f$ in eq. (7) or (9): The most immediate one is to look at the equilibrium PDF of the unconstrained system in eq. (23), before having eliminated the momenta from the description. The values that maximize this PDF are those that minimize the unconstrained Hamiltonian for each value of the rest of the coordinates, $u$, and all the momenta, $p$. This can be formally accounted for by stating that the functions $f$ take the values that make $H(u, d, p)$ a local minimum for each fixed $u$ and $p$, i.e.,

$$
H(u, f, p) \leq H(u, d, p), \forall d \in \mathcal{D}(f),
$$

where $\mathcal{D}(f)$ is a suitable open set in $\mathbb{R}^{L}$ containing the point $f$, and therefore the functions $f$ must satisfy

$$
\frac{\partial H}{\partial d^{I}}(u, f, p)=0, \quad I=K+1, \ldots, N,
$$

with the corresponding Hessian,

$$
\frac{\partial^{2} H}{\partial d^{I} \partial d^{J}}(u, f, p)
$$

being a positive definite $L \times L$ matrix.

Now, since the left-hand side of eq. (35) is precisely minus the right-hand side of the Hamilton equation in eq. $21 \mathrm{~b}$ for the time derivative $\dot{p}_{I}$ of the canonical momentum associated to $d^{I}$, we have that this kind of constraint is equivalent to asking that

$$
\dot{p}_{I}=0 \Rightarrow p_{I}(t)=p_{I}\left(t_{0}\right), \quad \forall t .
$$

Using the definition of the momenta in eq. 20 , this condition can be seen to define the following non-holonomic constraint involving the velocities:

$$
G_{I \mu}(q) \dot{q}^{\mu}=C_{I}
$$

being $C_{I}$ a constant number dependent on the initial conditions. In fact, if we look at the implicit definition of the functions $f$ in eq. (34) or $(35)$, we see that they must depend on the momenta, i.e., they are $f(u, p)$ and not $f(u)$, which already clashes with the holonomic scheme introduced in sec. 2.1.

Although some interpretation problems are associated to non-holonomic constraints [11, and they are known to not accept in general a closed Hamiltonian formalism 112 113114, they are perfectly valid in the algorithmic sense discussed before (i.e., as a tool to define the integration region in which the integrated quantity is non-negligible in equilibrium statistical mechanics). In fact, when their form is linear in the velocities, as it is the case of eq. (38), the corresponding equations of motion obtained from D'Alembert's principle are very simple [12113114, and, even if they cannot be expressed as the Hamilton equations of a given Hamiltonian function, it has been shown that their equilibrium distribution can be nevertheless computed [15]. In [49], for example, this type of flexible constraints are used to build a practical algorithm that is shown to be time-reversible and to present good energy conservation properties, whereas it is not symplectic; as expected from the non-Hamiltonian character of the dynamics. However, it remains to be proved, maybe using the techniques in [115, what is the equilibrium PDF produced by this dynamics. Since in this work we are interested in the statistical mechanics of constrained models, we prefer not 
to deal with this case related to the minimization of $H(q, p)$ until these fundamental problems are solved.

A different option to define the constrained subspace appears if we consider the frequent case of momenta-independent observables $O(q)$. In this situation, as we discussed before, the momenta can be 'integrated out' from the description, and equilibrium averages are computed as in eq. (30), using the marginal PDF defined in eq. (28). Following the same ideas that we applied in the momenta-dependent case, we see that the region of position space in which the integrated object in eq. 30 will be non-negligible is the one in which the equilibrium PDF is maximal; or, equivalently, that in which the quantity $F(u, d):=V(s, d)-T S^{\mathrm{k}}(u, d)$ defined in eq. (29) is made a local minimum for each value of the unconstrained coordinates $u$, i.e., we ask the functions $f$ that define the constrained subspace to satisfy

$$
F(u, f(u)) \leq F(u, d), \forall d \in \mathcal{D}(f(u)),
$$

where $\mathcal{D}(f(u))$ is again a suitable open set in $\mathbb{R}^{L}$ containing the point $f(u)$, and therefore we have that

$$
\frac{\partial F}{\partial d^{I}}(u, f(u)):=\frac{\partial V}{\partial d^{I}}(s, f(u))-T \frac{\partial S^{\mathrm{k}}}{\partial d^{I}}(u, f(u))=0, \quad I=K+1, \ldots, N,
$$

with the corresponding Hessian,

$$
\frac{\partial^{2} F}{\partial d^{I} \partial d^{J}}(u, f(u)),
$$

being a positive definite $L \times L$ matrix.

In contrast with the previous definition, this one (which has never been used in the literature as far as we are aware) does produce constraints that are holonomic, i.e., the functions $f$ that are now implicitly defined by the equations above depend only on position-like degrees of freedom (the unconstrained coordinates $u$ ), and the constrained subspace is now defined by the holonomic relations in eq. (7). Assuming D'Alembert's principle, these constraints produce a dynamics which is Hamiltonian, which is therefore amenable to symplectic integration methods, and whose equilibrium PDF can be easily computed using the same standard ideas that we develop in sec. 2.4.

In this work, however, for the sake of simplicity, we choose to define the flexible constrained subspace in a third, different way which is nevertheless related to both of the definitions just discussed. Simply stated, the function of the positions whose minimum we use to implicitly define the value $f$ of the constrained coordinates $d$ is neither the Hamiltonian $H(q, p)$ nor the free energy $F(q)$, but the potential energy $V(w)$, i.e., we ask that, for each fixed value $s$ of the unconstrained coordinates,

$$
V(s, f(s)) \leq V(s, d), \forall d \in \mathcal{D}(f(s)),
$$

where $\mathcal{D}(f(s))$ is a suitable open set in $\mathbb{R}^{L}$ containing the point $f(s)$, and we have that

$$
\frac{\partial V}{\partial d^{I}}(s, f(s))=0, \quad I=K+1, \ldots, N,
$$

with the corresponding Hessian,

$$
\mathcal{H}_{I J}^{\Sigma}(s):=\frac{\partial^{2} V}{\partial d^{I} \partial d^{J}}(s, f(s))
$$

being a positive definite $L \times L$ matrix.

Some points about this last definition are worth remarking before we move on: 

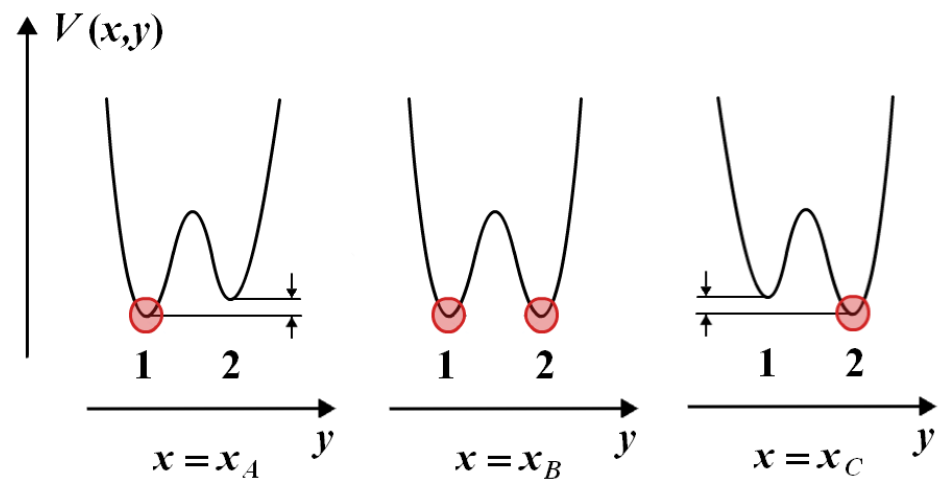

Fig. 2. Schematic depiction of an unphysical flip due to the use of the global minimum criterium for defining the constrained subspace. Three sections of the potential energy surface are shown at three different values of $x$. At $x_{A}$, the minimum 1 is the global one, whereas, at $x_{C}$, it is 2 . At $x_{B}$, the potential energy of 1 and 2 are the same, and the global minimum criterium becomes ambiguous.

- This choice, which is also used in refs. [54/50|79|69|67/88/89|73|41/82/83 and in all of the previous works by our group in this topic [48|55]116], can immediately be seen to come from either of the two previous definitions if some terms are neglected. In the momenta-dependent case, we need to neglect the derivative of the kinetic energy with respect to the constrained coordinates $d$ in eq. (35) to arrive to eq. (43); in the case in which the constraints are defined by minimizing the free energy $F(q)$, the derivative of $S^{\mathrm{k}}(q)$ in eq. 400 needs to be neglected to obtain the condition in eq. (43). Using that, for any $N \times N$ matrix $A(x)$ dependent on a parameter $x[53$,

$$
\frac{\partial \operatorname{det} A}{\partial x}=\operatorname{det} A \cdot \operatorname{tr}\left(A^{-1} \frac{\partial A}{\partial x}\right),
$$

and also that, trivially,

$$
A^{-1} A=\mathbb{I} \quad \Rightarrow \quad \operatorname{tr}\left(A^{-1} A\right)=N \quad \Rightarrow \quad \operatorname{tr}\left(\frac{\partial A^{-1}}{\partial x} A\right)+\operatorname{tr}\left(A^{-1} \frac{\partial A}{\partial x}\right)=0
$$

the reader can check that the mentioned condition on the kinetic energy implies the one on $S^{\mathrm{k}}(q)$ (as expected), but the reverse does not hold in general.

- As we will show in the following sections, if suitable internal coordinates are used [45], the expression of $\operatorname{det} G(q)$ as a function of the coordinates is actually very simple. Therefore, not only all the basic ideas in this work are applicable both to the definition of constraints using $F(q)$ and the one using $V(w)$, but it is also rather straightforward, technically speaking, to add the term associated to the minimization of $S^{\mathrm{k}}(q)$ to the mix. We plan to do this in future works.

- Since, as we mentioned, the potential energy function of molecular systems in the absence of external fields does not depend on the external coordinates, the constrained values $f$ defined in this last case do not depend on the whole set of unconstrained coordinates $u$, but only on the internal ones $s$; and the relevant constrained subspace is not $\mathcal{K}$ but $\Sigma$ (see sec. 2.1).

- For this last case, but also for the previous two, there are at least two reasons why the definition based on a local minima is more convenient that a possible definition based on a global one (such as the one we erroneously advocated in a previous work 
by some of us [48]): First, the problem of global minimization is a difficult one; for general functions it is known to be NP complete [117 118, and therefore, even if we wished for some reason to define the constrained subspace as the place where some function attains its global minimum, there is no guarantee that we will find the practical means to compute it. Secondly, such a definition would very likely cause unphysical effects: Imagine for the sake of simplicity that we have a system with two position-like degrees of freedom, denoted by $(x, y)$, and their respective momenta. If we identify the coordinate $y$ as 'stiff' and want to constraint it to its global minimum-energy value for each value of the unconstrained coordinate $x$, we could face the situation schematically depicted in fig. 2. In such a case, as we smoothly progress along the coordinate $x$, we can see that the global minimum definition might become ambiguous at some value $x=x_{B}$ and then the system can instantaneously change the value of its coordinate $y$ to one which is close in energy to the previous one but far away in conformational space. The flip is unphysical simply because, if the barrier separating the minima is high enough (something we shall need in order to consider $y$ stiff), then it will be a very unlikely event that an unconstrained trajectory actually performs the flip, and even if it does, it will not do it instantly (and with a discontinuity in the constrained coordinates) as in the flawed constrained approximation based on global minimization. This situation is not academic but actually very common when we have different molecular isomers, such as the cis and trans forms of the peptide bond in proteins [6].

Using the definition based on the minimization of $V(w)$ from now on, we can now relate the possible answers to question (a) in sec. 2.3.1 to the ideas introduced in this section: Following the reasoning that has led us to this point and which is behind the definition of the constrained subspace, a selected set of constrained coordinates, $d$, will be a priori a good choice [i.e., a good answer to (a)] if, for 'small' variations $\Delta d$ in $d$, it happens that $V(s, f(s)+\Delta d)-V(s, f(s)) \gg R T$. If this is so, the statistical weight, which is proportional to $\exp (-V(s, d) / R T)$, will become negligibly small as soon as we separate from the constrained subspace $\Sigma$ by any relevant amount. Albeit intuitively appealing (so much that we ourselves argued in this line in a previous work 48]), we will leave this argument at this point remarking that the scary quotes around 'small' actually hide real definition problems, as anyone can easily see if she considers that, to begin with, some internal coordinates have length units and some others are angles. We are at the moment pursuing a more mathematically involved and more rigorous definition of the properties that a proper 'stiff' constrained coordinate must have, but notice that this only affects the quantitative details pertaining what do we mean when we write 'small'; the basic, intuitive idea that the constrained coordinates are difficult to change due to energetic reasons is still valid.

Of course, the answer to question (b) discussed in this section, i.e., whether or not (or how much) the equilibrium values $f(s)$ of the constrained coordinates depend on the point in $\Sigma$ given by $s$, is also determined (by definition) by the nature of the potential energy used to model the system. Using Quantum Mechanics-based potentials, it has already been proved in small peptides that, for a given choice of constrained coordinates, their equilibrium values do depend on the conformation $s$ significantly 11948,120121. In this work, we will show en passant, as some previous works have already done 49,58,122 41,50, that the functions $f(s)$ also depend on $s$ in the case of the much simpler classical force fields [16 17|18 19|20|21] typically used for MD of proteins and nucleic acids. This is hardly surprising if we think that the force fields potential energy function typically has the following form:

$$
V_{\mathrm{FF}}(w):=\frac{1}{2} \sum_{\alpha=1}^{N_{l}} K_{l_{\alpha}}\left(l_{\alpha}-l_{\alpha}^{0}\right)^{2}+\frac{1}{2} \sum_{\alpha=1}^{N_{\theta}} K_{\theta_{\alpha}}\left(\theta_{\alpha}-\theta_{\alpha}^{0}\right)^{2}+V_{\mathrm{FF}}^{\mathrm{t}}\left(\phi_{\alpha}\right)+V_{\mathrm{FF}}^{\mathrm{lr}}(w)
$$


where $l_{\alpha}$ are bond lengths, $\theta_{\alpha}$ are bond angles, and $\phi_{\alpha}$ are dihedral angles. The integer $N_{l}$ is the number of bond lengths, $N_{\theta}$ the number of bond angles, and the quantities $K_{l_{\alpha}}, K_{\theta_{\alpha}}, l_{\alpha}^{0}$ and $\theta_{\alpha}^{0}$ are constant numbers. The term denoted by $V_{\mathrm{FF}}^{\mathrm{t}}\left(\phi_{\alpha}\right)$ is a commonly included torsional potential that depends only on the dihedral angles $\phi_{a}{ }^{5}$. On the other hand, $V_{\mathrm{FF}}^{\mathrm{lr}}$ normally comprises long-range interactions such as Coulomb or van der Waals, which involve $O\left(n^{2}\right)$ terms related to all possible atom pairs, each one of them depending on the difference of the atomic positions $\mathbf{r}_{\alpha}-\mathbf{r}_{\beta}$.

It is precisely this last dependence the one that couples all internal coordinates with one another, the one that makes force fields non-trivial, and the one that makes the minimum-energy values of, say, bond lengths and bond angles, not the constant numbers $l_{\alpha}^{0}$ and $\theta_{\alpha}^{0}$ in the force field but some $s$-dependent quantities.

Although it becomes clear that the most natural way of thinking about the physical origin of constraints presented in this section suggests that they must be (at least in some degree) flexible, i.e., that the functions $f(s)$ in eq. (9) must indeed depend on the unconstrained internal coordinates $s$, the most common approach (by far) in the com-

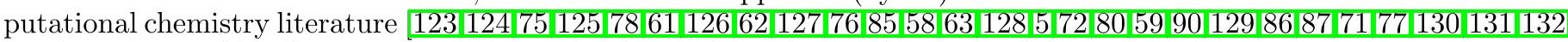
has been to consider them as constants, or hard; existing a few works, most of them recent, dealing with the flexible case 4913565164416966|50, including some previous works by us 4855116] (although it is worth remarking that the basic idea and the fact that it comes more naturally from physical considerations had been mentioned as early as 1969 by Gō and Scheraga [54]). This is partly due to numerical convenience but it also might be related to the great influence that classical force fields have had in the area. Indeed, if we take a look at the generic expression in eq. (47) of such an energy function, we see that the quantities, say, $l_{\alpha}^{0}$ and $\theta_{\alpha}^{0}$, appearing in the harmonic energy terms associated to bond lengths and bond angles, are readily available to be elected as the candidate constant numbers to which equate this coordinates should we want to consider them as 'stiff' and constrain them; even if they are not, as mentioned, the actual minimum-energy values of the bond lengths and bond angles in any given conformation of the molecule. If, instead of a force field, we consider a less explicit energy function, such as the one produced by the ground-state Born-Oppenheimer approximation for the electrons in quantum chemistry [23136], then no candidate number appears before our eyes as the constants to be used in the constrained model, and the flexible way of proceeding is even more natural.

A different but related misconception, probably also stemming from the prevalence of force fields in the literature about constraints, is the idea that the steeper the potential energy function is when the system leaves the constrained subspace, the better approximation the hard one becomes 73.72 . This is indeed true if the potential has a form such as the one in eq. (47), because, as the 'spring-constants' $K_{l_{\alpha}}$ and $K_{\theta_{\alpha}}$ get larger and larger, the associated harmonic terms overcome the long-range interactions, which were, as we argued, the ones responsible for the minimum-energy values of bond lengths and bond angles not being the constant numbers $l_{\alpha}^{0}$ and $\theta_{\alpha}^{0}$ but conformation-dependent quantities. However, this particular form of the potential energy function is not the only possible choice, and one could perfectly argue that harmonic terms such as the following ones, which contradict the previously stated argument,

$$
\frac{1}{2} \sum_{\alpha=1}^{N_{l}} K_{l_{\alpha}}\left(l_{\alpha}-l_{\alpha}^{0}(s)\right)^{2}+\frac{1}{2} \sum_{\alpha=1}^{N_{\theta}} K_{\theta_{\alpha}}\left(\theta_{\alpha}-\theta_{\alpha}^{0}(s)\right)^{2}
$$

5 The terms in $V_{\mathrm{FF}}^{\mathrm{t}}\left(\phi_{\alpha}\right)$ associated to 'soft' dihedrals, such as the Ramachandran angles in proteins, are typically trigonometric functions; whereas those associated to 'stiff' dihedrals, such as the peptide-bond dihedral angle $\omega$, are typically harmonic. 
may model the actual physics of the system more accurately in some cases. Indeed, using this kind of functional form, we can have infinitely steep potentials around the constrained subspace at the same time that the minimum-energy values of the constrained coordinates do depend on $s$.

In sec. 2.5 more information is provided about the use of particular energy functions in the literature and its influence on the flexible vs. hard issue, as well as on other approximations. We also point the reader to the discussion about the choice of coordinates in secs. 2.4.1 and sec. 2.4.3, which is very much related to the issue of the form of the potential energy function in the vicinity of the constrained subspace. Finally, in sec. 3, we analyze the difference between flexible and hard constraints in a simple methanol molecule, and some works are mentioned in which this same comparison has been performed in other practical cases.

\subsection{Statistical Mechanics models}

\subsubsection{Rigid model with flexible constraints}

The reasoning based in energetic considerations in the previous section justifies to substitute the fact that the system is more likely to evolve close to a given region of its internal space, namely, the internal constrained subspace $\Sigma$, by the approximation that it evolves only in that region.

When imposing constraints on a physical system to enforce this approximation, it is very common (specially in the theoretical physics literature 44.51 but also in computational chemistry works [5]3]) to assume not only that the constraints are exactly fulfilled at any given time, but also that D'Alembert's principle holds, i.e., that the force of constraint does no work (it is entirely orthogonal to the constrained subspace $\Sigma)$. It can be shown that, assuming these hypotheses, the dynamics of the system, described now in terms of the unconstrained coordinates $u$ (i.e., the external ones, $e$, plus the unconstrained internal ones, $s$ ) is the solution of the Hamilton equations associated to the following rigid Hamiltonian [84]:

$$
H_{\mathrm{r}}(u, \eta):=\frac{1}{2} \eta_{r} g^{r s}(u) \eta_{s}+V_{\Sigma}(s)
$$

where the restriction $V_{\Sigma}(s)$ of the potential energy $V(w)$ to the constrained subspace $\Sigma$ is defined as

$$
V_{\Sigma}(s):=V(s, f(s))
$$

being the functions $f(s)$ the ones that define the constraints in eq. (9) and whose origin has been discussed in the previous section. Of course, in the most general case, these functions do depend on $s$ and we are therefore working in the flexible setting.

Inserting these constraints into the kinetic energy in the unconstrained Lagrangian in eq. (17), as well as their time derivatives,

$$
\dot{d}^{I}=\frac{\partial f^{I}(s)}{\partial s^{i}} \dot{s}^{i}
$$

we obtain the rigid kinetic energy:

$$
K_{\mathrm{r}}(u, \dot{u}):=\frac{1}{2} \dot{u}^{r} g_{r s}(u) \dot{u}^{s}
$$

where $g_{r s}(u)$ is the induced mass-metric tensor (or pull-back), given by

$$
g_{r s}(u):=G_{r s}^{\mathcal{K}}(u)+\frac{\partial f^{I}(u)}{\partial u^{r}} G_{I s}^{\mathcal{K}}(u)+G_{r J}^{\mathcal{K}}(u) \frac{\partial f^{J}(u)}{\partial u^{s}}+\frac{\partial f^{I}(u)}{\partial u^{r}} G_{I J}^{\mathcal{K}}(u) \frac{\partial f^{J}(u)}{\partial u^{s}},
$$


being $G_{\mu \nu}^{\mathcal{K}}(u)$ simply the restriction of the whole-space MMT in eq. 18 to the constrained subspace $\mathcal{K}=\mathcal{E} \times \Sigma$, i.e.,

$$
G_{\mu \nu}^{\mathcal{K}}(u):=G_{\mu \nu}(e, s, f(s)) .
$$

Now eqs. 50) and (52) allows us to construct the rigid Lagrangian,

$$
L_{\mathrm{r}}(u, \dot{u}):=\frac{1}{2} \dot{u}^{r} g_{r s}(u) \dot{u}^{s}-V_{\Sigma}(s),
$$

which finally produces the rigid Hamiltonian in eq. 49 ) via the usual Legendre transform, defining the canonical conjugate momenta as

$$
\eta_{r}:=\frac{\partial L_{\mathrm{r}}}{\partial \dot{u}^{r}}=g_{r s}(u) \dot{u}^{s} .
$$

The constant-energy dynamics produced by the rigid Hamiltonian in eq. 499) (via the corresponding Hamilton equations) can be implemented in a computer using, for example, the algorithms described in refs. [50|4965]. If constant-temperature dynamics needs to be performed in this setting, one can use any of the methods available for hard rigid simulations 130/63/78 137, probably after some small adaptation to the flexible case [49|138]. See also refs. [109/110[139] in this special issue for more information about constrained simulations in ensembles other than the microcanonical one.

With regard to the associated equilibrium statistical mechanics, and making the same considerations we made for the unconstrained case in sec. 2.2. we can write the corresponding rigid partition function in the canonical ensemble as

$$
Z_{r}=\frac{\alpha_{\mathrm{QM}}}{h^{K}} \int e^{-\beta H_{\mathrm{r}}(u, \eta)} \mathrm{d} u \mathrm{~d} \eta .
$$

Hence, the equilibrium PDF is given by

$$
P_{\mathrm{r}}(u, \eta)=\frac{e^{-\beta H_{\mathrm{r}}(u, \eta)}}{\int e^{-\beta H_{\mathrm{r}}\left(u^{\prime}, \eta^{\prime}\right)} \mathrm{d} u^{\prime} \mathrm{d} \eta^{\prime}},
$$

which has the analogous meaning as the one attributed to eq. 23 in sec. 2.2 being the equilibrium average of any observable $O(u, \eta)$ :

$$
\langle O\rangle_{\mathrm{r}}=\int O(u, \eta) P_{\mathrm{r}}(u, \eta) \mathrm{d} u \mathrm{~d} \eta .
$$

Again, if we are interested in observables that are dependent only on the positions $u$, we can use eq. (25) to integrate out the momenta in eq. (57), yielding

$$
Z_{r}=\chi_{\mathrm{r}}(T) \int e^{-\beta\left[V_{\Sigma}(s)-T \frac{R}{2} \ln \operatorname{det} g(u)\right]} \mathrm{d} u,
$$

where

$$
\chi_{\mathrm{r}}(T):=\left(\frac{2 \pi}{\beta}\right)^{K / 2} \frac{\alpha_{\mathrm{QM}}}{h^{K}} .
$$

If we perform the same integration in the joint PDF in eq. (58), the factor $\chi_{\mathrm{r}}(T)$ cancels out and we arrive to the marginal equilibrium PDF in the space of the positions $u$ :

$$
P_{\mathrm{r}}(u)=\frac{e^{-\beta\left[V_{\Sigma}(s)-T \frac{R}{2} \ln \operatorname{det} g(u)\right]}}{\int e^{-\beta\left[V_{\Sigma}\left(s^{\prime}\right)-T \frac{R}{2} \ln \operatorname{det} g\left(u^{\prime}\right)\right]} \mathrm{d} u^{\prime}},
$$


being the equilibrium average of any momenta-independent observable $O(u)$

$$
\langle O\rangle_{\mathrm{r}}=\int O(u) P_{\mathrm{r}}(u) \mathrm{d} u .
$$

The same invariance of the potential energy of molecular systems with respect to the external coordinates, $e$, that we used in sec. 2.2 to integrate these degrees of freedom out is here applicable as well, and this is why we have written $V_{\Sigma}(s)$ and not $V_{\mathcal{K}}(u)$ in all the previous expressions. The induced MMT $g(u)$ in eq. (53) on the other hand, and in particular its determinant in eqs. 60 and $(62)$, does depend on the external coordinates. As in sec. 2.2 one can always formally integrate over the external coordinates in order to get to the corresponding marginal PDF in $\Sigma$, i.e., depending only on the unconstrained internal coordinates $s$. However, until recent, it was not clear if this process could be performed analytically for general flexible constraints, thus yielding manageable final expressions. In a previous work [55], we settled the issue proving that this is in fact possible and providing the exact analytical expressions to be used for the marginal PDF in $\Sigma$ (this case with constraints is much more involved that the unconstrained one mentioned in sec. 2.2. which had been proved long ago by Gō and Scheraga using a different development [72]).

We showed that the determinant of the reduced MMT $g(u)$ can be written as follows:

$$
\operatorname{det} g(u)=\sin ^{2} \theta \operatorname{det} g^{\prime}(s),
$$

where $\theta$ is one of the external Euler angles and the externals-independent matrix $g^{\prime}(s)$ is given by

$$
g^{\prime}(s)=\sum_{\alpha} m_{\alpha}\left(\begin{array}{cc|ccc}
\mathbb{I}^{(3)} & v\left(\mathcal{R}_{\alpha}^{\prime}\right) & \cdots & \frac{\partial \mathcal{R}_{\alpha}^{\prime}}{\partial s^{j}} & \cdots \\
v^{T}\left(\mathcal{R}_{\alpha}^{\prime}\right) & J\left(\mathcal{R}_{\alpha}^{\prime}\right) & \cdots & \frac{\partial \mathcal{R}_{\alpha}^{\prime}}{\partial s^{j}} \times \mathcal{R}_{\alpha}^{\prime} & \cdots \\
\hline \vdots & \vdots & & \vdots \\
\frac{\partial \mathcal{R}_{\alpha}^{\prime T}}{\partial s^{i}} & \left(\frac{\partial \mathcal{R}_{\alpha}^{\prime}}{\partial s^{i}} \times \mathcal{R}_{\alpha}^{\prime}\right. \\
\vdots & \cdots & \frac{\partial \mathcal{R}_{\alpha}^{\prime T}}{\partial s^{i}} \frac{\partial \mathcal{R}_{\alpha}^{\prime}}{\partial s^{j}} & \cdots \\
\vdots & & \vdots
\end{array}\right)
$$

where $\mathbb{I}^{(3)}$ is the $3 \times 3$ identity matrix, and the matrices $v$ and $J$ are defined as

$$
v\left(\mathcal{R}_{\alpha}^{\prime}\right):=\left(\begin{array}{ccc}
0 & -\mathcal{Z}_{\alpha}^{\prime} & \mathcal{Y}_{\alpha}^{\prime} \\
\mathcal{Z}_{\alpha}^{\prime} & 0 & -\mathcal{X}_{\alpha}^{\prime} \\
-\mathcal{Y}_{\alpha}^{\prime} & \mathcal{X}_{\alpha}^{\prime} & 0
\end{array}\right)
$$

and

$$
J\left(\boldsymbol{R}_{\alpha}^{\prime}\right):=\left(\begin{array}{ccc}
\mathcal{Y}_{\alpha}^{\prime 2}+\mathcal{Z}_{\alpha}^{\prime 2} & -\mathcal{X}_{\alpha}^{\prime} \mathcal{Y}_{\alpha}^{\prime} & -\mathcal{X}_{\alpha}^{\prime} \mathcal{Z}_{\alpha}^{\prime} \\
-\mathcal{X}_{\alpha}^{\prime} \mathcal{Y}_{\alpha}^{\prime} & \mathcal{X}_{\alpha}^{\prime 2}+\mathcal{Z}_{\alpha}^{\prime 2} & -\mathcal{Y}_{\alpha}^{\prime} \mathcal{Z}_{\alpha}^{\prime} \\
-\mathcal{X}_{\alpha}^{\prime} \mathcal{Z}_{\alpha}^{\prime} & -\mathcal{Y}_{\alpha}^{\prime} \mathcal{Z}_{\alpha}^{\prime} & \mathcal{X}_{\alpha}^{\prime 2}+\mathcal{Y}_{\alpha}^{\prime 2}
\end{array}\right)
$$

respectively, being $\mathcal{X}_{\alpha}^{\prime}, \mathcal{Y}_{\alpha}^{\prime}$ and $\mathcal{Z}_{\alpha}^{\prime}$ the three Euclidean components of $\mathcal{R}_{\alpha}^{\prime}$ (see sec. 2.1 for a precise definition of these quantities).

This result allows to factorize the exponential in eqs. 60 and 62 , and to perform a second integration to get rid of the uninteresting external coordinates $e$, thus arriving to the marginal equilibrium $\mathrm{PDF}$ in the space parameterized by the unconstrained 
internal coordinates $s$, i.e., $\Sigma$ :

$$
P_{\mathrm{r}}(s)=\frac{e^{-\beta F_{\mathrm{r}}(s)}}{\int e^{-\beta F_{\mathrm{r}}\left(s^{\prime}\right)} \mathrm{d} s^{\prime}},
$$

where we have defined

$$
\begin{aligned}
F_{\mathrm{r}}(s) & :=V_{\Sigma}(s)-T S_{\mathrm{r}}^{\mathrm{k}}(s), \\
S_{\mathrm{r}}^{\mathrm{k}}(s) & :=\frac{R}{2} \ln \operatorname{det} g^{\prime}(s) .
\end{aligned}
$$

Again, the notation in the equations above is intentional, and we regard $F_{\mathrm{r}}(s)$ as a free or effective energy, and $S_{\mathrm{r}}^{\mathrm{k}}(s)$ as a sort of a kinetic entropy.

Using $P_{\mathrm{r}}(s)$ above, the equilibrium average of any observable $O(s)$ that depends only on $s$ can be calculated as

$$
\langle O\rangle_{\mathrm{r}}=\int O(s) P_{\mathrm{r}}(s) \mathrm{d} s .
$$

Finally, let us remark that the derivation in this section is equivalent to the ones in refs. $63|31| 69|88| 89|79| 128|82| 83 \mid 131$, being the only difference that, in those works, different generalized coordinates $\tilde{q}$ are chosen in such a way that the constraint functions in eq. (8),

$$
\sigma^{I}(w):=d^{I}-f^{I}(s), \quad I=K+1, \ldots, N,
$$

are among them. Therefore, in these new coordinates $\tilde{q}$, the constraints and their time derivatives are not expressed as in eqs. (9) and (51), but simply as

$$
\begin{array}{ll}
\tilde{d}^{I}=0, & I=K+1, \ldots, N, \\
\dot{\tilde{d}}^{I}=0, & I=K+1, \ldots, N .
\end{array}
$$

Despite the earned simplifications that this choice brings, we have preferred to use coordinates which do not include the functions $\sigma$ among them because typical internal coordinates used in molecular simulations in fact do not include these functions [45, and therefore we can more easily relate to the chemically intuitive quantities represented by them (bond lengths, bond angles, etc.) in the formalism. However, since many works use the modified coordinates $\tilde{q}$, we will make a stop now and then to mention how this choice affects the final expressions and conclusions in this work.

The transformation to the new coordinates is actually very simple:

$$
\begin{aligned}
& \tilde{u}^{r}=\tilde{U}^{r}(q):=u^{r}, \quad r=1, \ldots, K, \\
& \tilde{d}^{I}=\tilde{D}^{I}(q):=d^{I}-f^{I}(s), \quad I=K+1, \ldots, N,
\end{aligned}
$$

and so it is its inverse:

$$
\begin{aligned}
& u^{r}=U^{r}(\tilde{q}):=\tilde{u}^{r}, \quad r=1, \ldots, K, \\
& d^{I}=D^{I}(\tilde{q}):=\tilde{d}^{I}+f^{I}(\tilde{s}), \quad I=K+1, \ldots, N .
\end{aligned}
$$

We will see in what follows that the fact that this transformation affects only the definition of the constrained coordinates, $d$, produces remarkable properties and very simple rules for most of the transformed objects. 
This can already be seen in the structure of the Jacobian matrix:

$$
\left(J_{\nu}^{\mu}\right):=\left(\frac{\partial Q^{\mu}(\tilde{q})}{\partial \tilde{q}^{\nu}}\right)=\left(\begin{array}{ccc|ccc}
1 & & 0 & & \\
& \ddots & & & 0 & \\
0 & & 1 & & \\
\hline & \vdots & & & 0 \\
& \frac{\partial f^{I}(\tilde{s})}{\partial \tilde{s}^{i}} & \cdots & & \ddots & \\
\vdots & & 0 & & 1
\end{array}\right)
$$

and its inverse:

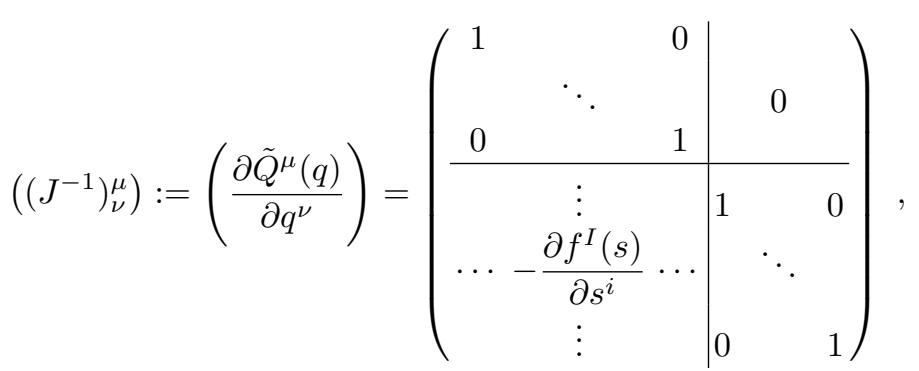

both of which have unit determinant: $\operatorname{det} J=\operatorname{det} J^{-1}=1$.

It is also worth mentioning that, if the constraints are defined as hard (see the next section), then the $\sigma$ functions in eq. (71) become

$$
\sigma^{I}(w):=d^{I}-d_{0}^{I}, \quad I=K+1, \ldots, N
$$

being $d_{0}^{I}$ constant numbers, and in such a case the simplicity of the change of coordinates is even higher, being the Jacobian and its inverse in the expressions above both exactly equal to the identity matrix.

In any case, either if the generalized velocities are related as in eq. (51) or if they are zero as in eq. (72b) and in refs. 63/31698889791288283131, it is convenient to bear in mind that momenta and velocities are only proportional when the matrix in the kinetic energy is diagonal (e.g., in Euclidean coordinates), and the statement sometimes found in the literature 708573766128133134 about the cancelation of the momenta associated to the constrained coordinates is wrong in general.

Regarding the calculations in this section, the use of the modified coordinates $\tilde{q}$ leads to a simpler expression for the induced MMT in eq. (53):

$$
\tilde{g}_{r s}(\tilde{u})=\tilde{G}_{r s}^{\mathcal{K}}(\tilde{u})
$$

i.e., it is simply the unconstrained-unconstrained block of the whole-space MMT $\tilde{G}$ evaluated in the constrained subspace (see sec. 2.5 for the implications of this in the calculation of Fixman's potential). 
Moreover, if we use eq. 109 for the transformation of the whole-space MMT $\tilde{G}$ together with the change of coordinates in eq. 74 , we can prove that

$$
\begin{aligned}
\tilde{g}_{r s}(\tilde{u})= & \tilde{G}_{r s}^{\mathcal{K}}(\tilde{u}):=\tilde{G}_{r s}(\tilde{u}, 0) \\
= & G_{r s}(Q(\tilde{u}, 0))+\frac{\partial f^{I}(\tilde{u})}{\partial \tilde{u}^{r}} G_{I s}(Q(\tilde{u}, 0))+G_{r J}(Q(\tilde{u}, 0)) \frac{\partial f^{J}(\tilde{u})}{\partial \tilde{u}^{s}} \\
& +\frac{\partial f^{I}(\tilde{u})}{\partial \tilde{u}^{r}} G_{I J}(Q(\tilde{u}, 0)) \frac{\partial f^{J}(\tilde{u})}{\partial \tilde{u}^{s}} \\
= & G_{r s}(\tilde{u}, f(\tilde{u}))+\frac{\partial f^{I}(\tilde{u})}{\partial \tilde{u}^{r}} G_{I s}(\tilde{u}, f(\tilde{u}))+G_{r J}(\tilde{u}, f(\tilde{u})) \frac{\partial f^{J}(\tilde{u})}{\partial \tilde{u}^{s}} \\
& +\frac{\partial f^{I}(\tilde{u})}{\partial \tilde{u}^{r}} G_{I J}(\tilde{u}, f(\tilde{u})) \frac{\partial f^{J}(\tilde{u})}{\partial \tilde{u}^{s}}=g_{r s}(\tilde{u}),
\end{aligned}
$$

i.e., the induced MMT is not modified upon the change of coordinates from $q$ to $\tilde{q}$; it is the same as the original one component-wise.

This directly implies that the calculations associated to the factorization and elimination of the external coordinates in ref. [55, which led us to expressions (64), (65), and related quantities, still apply in the new modified coordinates. The only change to be done is to place a 'tilde' over the appropriate symbols.

\subsubsection{Rigid model with hard constraints}

As we mentioned in sec. 2.3.2 the most common constrained dynamics considered in the literature is not the rigid one with flexible constraints described in the previous section, but the one with hard constraints. This option, which is numerically simpler, and which can be accurate enough for certain applications, is described in the following paragraphs.

The key difference between the two models is that, in the hard case, the constrained coordinates $d$ are not equal to some functions $f(s)$ of the unconstrained internal coordinates, as expressed in eq. (9), but to some constant numbers $d_{0}$ :

$$
d^{I}=d_{0}^{I}, \quad I=K+1, \ldots, N .
$$

This makes all partial derivatives in eqs. (51) and (53) zero, and converts the induced MMT $g(u)$ simply in the unconstrained-unconstrained sub-block of the restriction to $\mathcal{K}$ of the whole-space MMT, i.e.,

$$
\bar{g}_{r s}(u)=\bar{G}_{r s}^{\mathcal{K}}(u):=G_{r s}\left(e, s, d_{0}\right),
$$

where we have used an over-bar to denote hard objects. This fact has important implications regarding the Fixman's compensating potential, introduced in sec. 2.5. Also notice, that, contrarily to eq. (78), which only held in the flexible case if the modified coordinates $\tilde{q}$ were used, we now have this property in the original molecular coordinates $q$.

Another, more subtle consequence of the use of hard constraints affects the calculations of the partial derivatives $\partial \mathcal{R}_{\alpha}^{\prime} / \partial s^{i}$, which are a key ingredient of the matrix $g^{\prime}(s)$ in eq. 65. If we realize that the functions $\boldsymbol{\mathcal { R }}_{\alpha}^{\prime}(s)$ are implicitly defined in eq. 10 as

$$
\boldsymbol{\mathcal { R }}_{\alpha}^{\prime}(s):=\mathbf{R}_{\alpha}^{\prime}(s, f(s)),
$$

the sought derivatives can be computed using

$$
\frac{\partial \boldsymbol{\mathcal { R }}_{\alpha}^{\prime}}{\partial s^{i}}(s)=\frac{\partial \mathbf{R}_{\alpha}^{\prime}}{\partial s^{i}}(s, f(s))+\frac{\partial \mathbf{R}_{\alpha}^{\prime}}{\partial d^{I}}(s, f(s)) \frac{\partial f^{I}}{\partial s^{i}}(s) .
$$


In the flexible case, all terms in the sum are in principle non-zero, and the fact that the derivatives $\partial f^{I} / \partial s^{i}$ must be typically found numerically requires the use of complicated algorithms to obtain this quantities (see sec. 3 and ref. [16]). In the hard case, on the other hand, all these derivatives are zero and eq. 83 reduces to

$$
\frac{\partial \boldsymbol{\mathcal { R }}_{\alpha}^{\prime}}{\partial s^{i}}(s)=\frac{\partial \mathbf{R}_{\alpha}^{\prime}}{\partial s^{i}}\left(s, d_{0}\right)
$$

which contains only explicit derivatives which can typically be computed analytically. The efficient calculation of the MMT, MMT determinants, and related quantities in refs. 87/124]127 128/86 71/77], where they consider only hard constraints, is implicitly based on this fact.

Apart from these (important) differences, the dynamics of the rigid model with hard constraints is derived following the same steps we took in the previous section, and the rigid Hamiltonian in eq. 490 is still applicable to this case as long as we compute the induced MMT as indicated in eq. (81). This dynamics can be implemented in a computer using many different algorithms (see refs. 1306378, and references therein; and also [140] in this special issue), and, again, under the usual assumptions of ergodicity and equal a priori probabilities, its statistical mechanics equilibrium can be described in a formally identical way to the flexible case, via eqs. (68), (69a) and $(69 \mathrm{~b})$.

Despite this formal identity, however, it has been shown that the flexible and hard dynamics produce different physical results (see refs. 64496914150 and also sec. 3), and indeed in sec. 2.3.2 we built a strong case for considering the latter as an approximation of the former. In sec. 3, we will additionally illustrate how this difference in the dynamics produce measurable discrepancies at the level of equilibrium statistical mechanics in a methanol molecule.

\subsubsection{Stiff model with flexible constraints}

In the previous two sections, we discussed the dynamics as well as the canonical equilibrium of the so-called rigid model, in which not only the constraints are assumed to hold exactly but also D'Alembert's principle, which hypothesizes that the components of the forces of constraint tangent to the constrained subspace $\Sigma$ are zero 4484 .

Nevertheless, if we accept the unconstrained dynamics as the reference against which to assess the accuracy of any approximate model (see the discussion supporting this choice in sec. 2.3.2, we must realize that, if the system is in contact with a thermal bath at temperature $T$, even if there exist strong forces that drive it to the constrained subspace $\Sigma$, there also exist random fluctuations that will inevitably take the system away from this region. Moreover, even in the case in which we can assume that the potential energy outside $\Sigma$ tends to infinity (a case in which the constraints hold exactly by simple energy conservation arguments [142]), it can be proved that the force on $\Sigma$ resulting from this limit procedure not only consists of the orthogonal component needed to keep the system in the constrained subspace, but also of a nonzero tangent component that must be added to the expected gradient of $V_{\Sigma}(s)$, i.e., D'Alembert's principle, even in the infinitely steep confining potential case, is not satisfied in general 84,96,40 106 143 81.

These two in general violated assumptions confirm what we already suspected: that the rigid dynamics and statistical mechanics equilibrium are just an approximation of the real, unconstrained ones (even if we use the more physically sound flexible constraints). Therefore, if the rigid dynamics is to be used in practical simulations in 
order to gain the advantages discussed in sec. 1, it becomes necessary to assess how much error these approximations introduce.

Of course, in order to compare the rigid statistical mechanics equilibrium PDF $P_{\mathrm{r}}(s)$ in eq. (68) to the unconstrained one in $P(w)$ in eq. 32 (or the free energies in their respective exponents), we should arrive to some objects that are defined on the same space, being the most natural candidates $P_{\mathrm{r}}(s)$ itself and the marginal unconstrained PDF in the space, $\Sigma$, spanned by the unconstrained internal coordinates $s$. This last function is obtained through the integration of the constrained coordinates $d$ as usual:

$$
P(s):=\int P(w) \mathrm{d} d .
$$

However, in the general case this is only formal, i.e., for a general potential $V(w)$ and general curvilinear coordinates $q$, this integral cannot be calculated analytically. One of the available options is to compute it numerically, with the help of methods for calculating free energy differences 144311453233. In this work, on the other hand, we have chosen to develop a statistical mechanics model, termed stiff, whose effective free energy in $\Sigma$ can be analytically obtained and compared to the rigid one in a more direct and insightful way than the result of numerically performing the integral in eq. (85), at the same time that it could be considered an approximation to the unconstrained equilibrium containing weaker assumptions than the rigid one. Contrarily to the rigid model, the stiff one does account for the statistical weights of the conformations close to (but out of) $\Sigma$, at the same time that it allows for the existence of velocities that are orthogonal to the constrained subspace. Also, in the derivation of the stiff model, D'Alembert's principle is never invoked. These arguments, together with the knowledge of the controlled steps that take us from the unconstrained case to the stiff model, suggest that one could expect the stiff equilibrium to be closer to the unconstrained one than the rigid equilibrium not only conceptually but also in quantitative terms. Although in this article we will assume this to be true and the stiff model will be used as the reference against which to assess the accuracy of any constrained model, numerical confirmation will be pursued in future works.

The first step in the derivation of the stiff model consists of Taylor expanding the potential energy $V(s, d)$ at a fixed point $s$, in terms of the constrained coordinates $d$, around the point $d=f(s)$ :

$$
\begin{aligned}
V(s, d) & =V(s, f(s))+\left[\frac{\partial V}{\partial d^{I}}(s, f(s))\right]\left(d^{I}-f^{I}(s)\right) \\
& +\frac{1}{2}\left[\frac{\partial^{2} V}{\partial d^{I} \partial d^{J}}(s, f(s))\right]\left(d^{I}-f^{I}(s)\right)\left(d^{J}-f^{J}(s)\right)+O\left((d-f(s))^{3}\right),
\end{aligned}
$$

or, realizing that to evaluate in $(s, f(s))$ is to evaluate in the internal constrained subspace $\Sigma$,

$$
\begin{aligned}
V(s, d) & =V_{\Sigma}(s)+\partial_{I} V_{\Sigma}(s)\left(d^{I}-f^{I}(s)\right) \\
& +\frac{1}{2} \mathcal{H}_{I J}^{\Sigma}(s)\left(d^{I}-f^{I}(s)\right)\left(d^{J}-f^{J}(s)\right)+O\left((d-f(s))^{3}\right),
\end{aligned}
$$

where we have used the notation for the constrained Hessian in eq. (44), and we have introduced the notation $\partial_{I} V_{\Sigma}(s)$ for the gradient of $V(w)$ restricted to $\Sigma$.

The same expansion can be performed on $S^{\mathrm{k}}(w)$, yielding

$$
\begin{aligned}
S^{\mathrm{k}}(s, d) & =S_{\Sigma}^{\mathrm{k}}(s)+\partial_{I} S_{\Sigma}^{\mathrm{k}}(s)\left(d^{I}-f^{I}(s)\right) \\
& +\frac{1}{2} \partial_{I J}^{2} S_{\Sigma}^{\mathrm{k}}(s)\left(d^{I}-f^{I}(s)\right)\left(d^{J}-f^{J}(s)\right)+O\left((d-f(s))^{3}\right),
\end{aligned}
$$


and both of them can be introduced into the integral that appear in the definition of the marginal PDF in eq. 85):

$$
P(s):=\int P(w) \mathrm{d} d=\frac{\int e^{-\beta\left[V(w)-T S^{\mathrm{k}}(w)\right]} \mathrm{d} d}{\int e^{-\beta\left[V\left(w^{\prime}\right)-T S^{\mathrm{k}}\left(w^{\prime}\right)\right]} \mathrm{d} s^{\prime} \mathrm{d} d^{\prime}} .
$$

Note that expanding the term associated to the determinant of the whole-space MMT $\operatorname{det} G$ in the marginal PDF in positions space is in principle more accurate that the procedure followed in refs. [79 88 8963|128|5|90129 82 83146] or in our own work in ref. 48, where the dependence of $\operatorname{det} G$ on the constrained coordinates $d$ is simply neglected, evaluating this determinant directly in $d=f(s)$ and thus effectively truncating the expansion in eq. (88) at zero order (often implicitly). In fact, this point and the inclusion of flexible constraints in the mix are the only issues that make the formalism in this work more general than the ones in refs. 487988898283 .

Now, if we assume that the constrained coordinates are indeed 'stiff', i.e., that, as mentioned in sec. 2.3.2 for 'small' variations $\Delta d$ in $d$, we have $V(s, f(s)+\Delta d)-$ $V(s, f(s)) \gg R T$, then the quantities under the integral signs, which are proportional to $e^{-V(s, d) / R T}$, will become negligibly small as soon as we separate from the constrained subspace $\Sigma$ by any relevant amount. Moreover, we also know that, close to $\Sigma$, the Taylor expansions in eqs. (87) and $(88)$ can be truncated at low order. Therefore, for each fixed value of the unconstrained coordinates $s$, we can substitute the potential energy and the kinetic entropy terms by their respective low-order Taylor expansions. Also note that this very same argument can be used if we had chosen to define $\Sigma$ through the minimization of $F(q)$ instead of $V(w)$.

In principle, there is no reason to truncate at different orders the potential energy $V(s, d)$ and the kinetic entropy $S^{\mathrm{k}}(s, d)$.

If we truncate both expansions at order zero, we simply have that the exponential does not depend on the constrained coordinates, and the integral $\int 1 \mathrm{~d} d$ cancels out in the numerator and the denominator of eq. $(32)$, yielding the $(0,0)$-stiff PDF in $\Sigma$ :

$$
P_{\mathrm{s}}^{(0,0)}(s)=\frac{e^{-\beta F_{\mathrm{s}}^{(0,0)}(s)}}{\int e^{-\beta F_{\mathrm{s}}^{(0,0)}\left(s^{\prime}\right)} \mathrm{d} s^{\prime}},
$$

where we have defined

$$
\begin{aligned}
F_{\mathrm{s}}^{(0,0)}(s) & :=V_{\Sigma}(s)-T S_{\Sigma}^{\mathrm{k}}(s), \\
S_{\Sigma}^{\mathrm{k}}(s) & :=\frac{R}{2} \ln \operatorname{det} G_{\Sigma}^{\prime}(s),
\end{aligned}
$$

being $G_{\Sigma}^{\prime}(s)$ the restriction to $\Sigma$ of the matrix $G^{\prime}(w)$.

Although this statistical mechanics model is well-defined and may be certainly considered for some applications (in sec. 2.5 we mention a number of works in which it has been implicitly used), two issues lead to try to improve it: On the one hand, the truncation of the Taylor expansions at order zero means that we are only looking at points exactly on $\Sigma$, and not 'close' to it, which was one of the weaknesses, we argued, of the rigid model. On the other hand, the function we are substituting $V(s, d)$ with, i.e., $V_{\Sigma}(s)$, does not have the important property of becoming very large when the constrained coordinates get away from $\Sigma$ (because it does not depend on them!). The fact that we could obtain a finite $\operatorname{PDF}, P_{\mathrm{s}}^{(0,0)}(s)$, is due to the cancellation of an infinite quantity ${ }^{6} \int 1 \mathrm{~d} d$, in the numerator and the denominator. However, if

\footnotetext{
6 At least if any bond length is included among the constrained coordinates, which is always the case in practical applications.
} 
we consider the partition function of the system after performing the substitution, $Z=\int e^{-\beta\left(V_{\Sigma}(s)-T S_{\Sigma}^{\mathrm{k}}(s)\right)} \mathrm{d} s \mathrm{~d} d$, we see that it is infinite, which is certainly a warning and probably a problem.

If we truncate both expansions at the first order, and we assume that we are dealing with flexible constraints defined by the functions $f(s)$ taking the values of the constrained coordinates that minimize the potential energy at point $s$, then the first derivatives $\partial_{I} V_{\Sigma}(s)$ are zero, and the leading order in the expansion of $V(s, d)$ is again $V_{\Sigma}(s)$. Therefore, for the $(1,1)$-stiff model, the exponent in the integral is proportional to $V_{\Sigma}(s)-T S_{\Sigma}^{\mathrm{k}}(s)-T \partial_{I} S_{\Sigma}^{\mathrm{k}}(s)\left(d^{I}-f^{I}(s)\right)$, and, since the sign of the terms $\partial_{I} S_{\Sigma}^{\mathrm{k}}(s)$ can be anyone, there is no reason for the integral on the constrained coordinates to be convergent. If we considered the definition of $\Sigma$ involving the minimization not of $V(w)$ but of $F(q)$, the situation is slightly different but simple, since in this case $\partial_{I} V_{\Sigma}(s)-T \partial_{I} S_{\Sigma}^{\mathrm{k}}(s)=0$, and the $(1,1)$-stiff model would be equivalent to the $(0,0)$ one.

It becomes then clear that the second order terms must be included in the model if we want to work with finite quantities. Truncating both expansions at second order, and using eq. (25), we find the most general expression for the integral over the constrained coordinates that will be considered in this work:

$$
\begin{aligned}
& \int \exp \left[-\beta\left(V_{\Sigma}-T S_{\Sigma}^{\mathrm{k}}+\left(\partial_{I} V_{\Sigma}-T \partial_{I} S_{\Sigma}^{\mathrm{k}}\right)\left(d^{I}-f^{I}\right)\right.\right. \\
& \left.\left.\quad+\frac{1}{2}\left(d^{I}-f^{I}\right) A_{I J}\left(d^{J}-f^{J}\right)\right)\right] \mathrm{d} d \\
& =\left(\frac{2 \pi}{\beta}\right)^{\frac{L}{2}} \operatorname{det}^{-\frac{1}{2}} A \exp \left[\frac{\beta}{2}\left(\partial_{I} V_{\Sigma}-T \partial_{I} S_{\Sigma}^{\mathrm{k}}\right) A^{I J}\left(\partial_{J} V_{\Sigma}-T \partial_{J} S_{\Sigma}^{\mathrm{k}}\right)\right],
\end{aligned}
$$

where we have omitted the dependence on $s$, and we have defined

$$
A_{I J}:=\mathcal{H}_{I J}^{\Sigma}-T \partial_{I J}^{2} S_{\Sigma}^{\mathrm{k}},
$$

in order to lighten the notation. We have also approximated the different ranges of integration of the constrained coordinates all by the $(-\infty, \infty)$ range (notice that bond lengths range from 0 to $\infty$, bond angles from 0 to $\pi$, etc.). The reason why this is expected to be a good approximation is the same that supports the rest of the calculations, namely, that the integrated quantity is only non-negligible close to the constrained subspace $\Sigma$, where the constrained coordinates are typically far away from the true integration limits.

Despite this formal result, one needs to notice that, for the above integral to exist, the matrix $A$ has to be positive definite. If we had chosen the definition of the constrained subspace based on the minimization of $F(q)$, this property would be satisfied, since $A$ is precisely the Hessian of $F(q)$ at a minimum. In the case treated here, however, the positive definiteness of $A$ is not guaranteed. If we are in a minimum of the potential energy, the constrained Hessian $\mathcal{H}_{I J}^{\Sigma}$ in eq. 440 is indeed positive definite, but the presence of the term $-T \partial_{I J}^{2} S_{\Sigma}^{\mathrm{k}}$ spoils this property, in general, for the matrix $A$. If such a thing happens, or even if it is 'close' to happening (for example, if the smallest eigenvalue of $A$ becomes too close to zero), we must consider the possibility of moving to the model based on the minimization of $F(q)$.

Another issue that may cause the eigenvalues of the matrix $A$ to be small and thus render the stiff approximation questionable is that the coordinates we have selected as constrained may actually be a bad choice. Indeed, if the statistical weights of the conformations do not become significantly small as we move away from $\Sigma$ at second order in the unconstrained free energy $F(w):=V(w)-T S^{\mathrm{k}}(w)$, then we have little reasons to believe that the situation might change if we include higher orders. Hence, 
although the $(2,2)$-stiff model might not be a very good approximation at every point $s$, we should see the positive definiteness of the matrix $A$ in eq. (93) and the size of its eigenvalues as a consistency check, and regard those points at which these conditions fail as points in which the $(2,2)$-stiff model is a bad approximation of the unconstrained equilibrium, and the constrained coordinates are probably not 'stiff' enough. Although we have not found conformations in which $A$ (or $\mathcal{H}$; see below) contains negative eigenvalues in the practical example in sec. 3 , this is probably so due to the small size of the molecule studied. For larger systems, we have already seen some preliminary indications that these matrices might in fact become not positive definite depending on the chosen constrained coordinates.

Now, using eq. (92) and recalling that the first derivatives of the potential energy restricted to $\Sigma$ are zero in the chosen model, we have that the (2,2)-stiff PDF in $\Sigma$ is given by

$$
P_{\mathrm{s}}^{(2,2)}(s)=\frac{e^{-\beta F_{\mathrm{s}}^{(2,2)}(s)}}{\int e^{-\beta F_{\mathrm{s}}^{(2,2)}\left(s^{\prime}\right)} \mathrm{d} s^{\prime}},
$$

with

$$
F_{\mathrm{s}}^{(2,2)}(s):=V_{\Sigma}(s)-T S_{\Sigma}^{\mathrm{k}}(s)-T S_{A}^{\mathrm{c}}(s)-\frac{T^{2}}{2} \partial_{I} S_{\Sigma}^{\mathrm{k}}(s) A^{I J}(s) \partial_{J} S_{\Sigma}^{\mathrm{k}}(s)
$$

being $A^{I J}$ the entries of $A^{-1}$ and

$$
S_{A}^{\mathrm{c}}(s):=-\frac{R}{2} \ln \operatorname{det} A(s)
$$

where the letter ' $c$ ' in the new entropy stands for conformational, in reference to the fact that it appears as the result of eliminating some positions, and not momenta, being reminiscent of the conformational or configurational entropies appearing in quasi-harmonic analysis [1479091. The only difference that we would find if we chose the definition based on the minimization of $F(q)$ is that the last term in eq. (95) would not appear. As we mentioned, the $(2,2)$-stiff model is the most general stiff model in this work and it has never been used before in the literature as far as we know.

The calculation of $\mathcal{H}_{\Sigma}(s)$, which appears in $A(s)$, can be performed in many different ways depending on the potential energy we are dealing with (the computation of the other part of $A$ is described later). The calculation of $\operatorname{det} G_{\Sigma}^{\prime}(s)$, on the other hand, is entirely 'geometric', depending only on the scheme of internal coordinates chosen to describe the system (assuming that the functions $f(s)$ have already been calculated). The way to compute $\operatorname{det} G^{\prime}(w)$ analytically has been described before, using the analogue of eq. 65 but without constraints. However, if a specific set of internal coordinates is chosen, one can arrive to a much simpler and more explicit expression. In our work in ref. [55, we showed that, if the SASMIC internal coordinates 45] for general branched molecules are used (a similar result will hold for any well-designed scheme of internal coordinates), we have that

$$
\operatorname{det} G^{\prime}(w)=\left(\prod_{\alpha=1}^{n} m_{\alpha}^{3}\right)\left(\prod_{\alpha=2}^{n} l_{\alpha}^{4}\right)\left(\prod_{\alpha=3}^{n} \sin ^{2} \theta_{\alpha}\right),
$$

where $l_{\alpha}$ are the bond lengths, and $\theta_{\alpha}$ the bond angles associated to atom $\alpha$. The masses can be eliminated, since they come out of the logarithm as an additive term, which represents a constant change of reference in the free energy, and has no effect whatsoever in the equilibrium PDF. Gō and Scheraga also proved this result long ago using different mathematics [72]. 
This expression, together with the additivity of the logarithm, not only helps to easily calculate $S^{\mathrm{k}}(w)$ [and therefore its restriction $S_{\Sigma}^{\mathrm{k}}(s)$ ], but also its derivatives with respect to any internal coordinate depending on its type:

$$
\begin{aligned}
& \frac{\partial S^{\mathrm{k}}}{\partial l_{\alpha}}(w)=\frac{2 R}{l_{\alpha}}, \\
& \frac{\partial S^{\mathrm{k}}}{\partial \theta_{\alpha}}(w)=R \operatorname{cotg} \theta_{\alpha}, \\
& \frac{\partial S^{\mathrm{k}}}{\partial \phi_{\alpha}}(w)=0
\end{aligned}
$$

where $\phi_{\alpha}$ denotes the dihedral angle associated to atom $\alpha$.

Using these results, we can rewrite eq. (95) as

$$
F_{\mathrm{s}}^{(2,2)}(s):=V_{\Sigma}(s)-T S_{\Sigma}^{\mathrm{k}}(s)-T S_{A}^{\mathrm{c}}(s)+U_{\mathrm{s}}^{(2)}(s)
$$

with

$$
U_{\mathrm{s}}^{(2)}(s):=-\frac{1}{2 \beta^{2}} D\left(f^{I}(s)\right) A^{I J}(s) D\left(f^{J}(s)\right),
$$

being $D\left(d^{I}\right)$ the function defined as

$$
D\left(d^{I}\right):=\left\{\begin{array}{ll}
2 / d^{I} & \text { if } d^{I} \text { is a bond length } \\
\operatorname{cotg} d^{I} & \text { if } d^{I} \text { is a bond angle } \\
0 & \text { if } d^{I} \text { is a dihedral angle }
\end{array} .\right.
$$

The entries, $\partial_{I J}^{2} S^{\mathrm{k}}(w)$, of the matrix whose restriction to $\Sigma$ appears in the definition of $A$ in eq. 93 can also be easily computed, yielding

$$
T \partial_{I J}^{2} S^{\mathrm{k}}(w)=-\frac{1}{\beta} \delta_{I J} D_{2}\left(d^{I}\right)
$$

where $\delta_{I J}$ is the Kronecker's delta, and $D_{2}\left(d^{I}\right)$ is the function defined as

$$
D_{2}\left(d^{I}\right):=\left\{\begin{array}{ll}
2 /\left(d^{I}\right)^{2} & \text { if } d^{I} \text { is a bond length } \\
1 / \sin ^{2} d^{I} & \text { if } d^{I} \text { is a bond angle } \\
0 & \text { if } d^{I} \text { is a dihedral angle }
\end{array},\right.
$$

allowing to rewrite eq. (93) as

$$
A_{I J}(s):=\mathcal{H}_{I J}^{\Sigma}(s)+\frac{1}{\beta} \delta_{I J} D_{2}\left(f^{I}(s)\right)
$$

Note that both $D\left(d^{I}\right)$ and $D_{2}\left(d^{I}\right)$ are singular only in points that have little physical sense and that will be never reached in a practical simulation: $l_{\alpha}=0$ or $\theta_{\alpha}=0, \pi$. Not only the energy will be very large (even infinite) at these points, but also the change of coordinates in eq. (1) from the Euclidean to the curvilinear coordinates becomes singular.

Apart from the $(2,2)$-stiff model, we can also define two other models which yield convergent integrals for any point $s$. However, they are derived truncating the potential energy and the kinetic entropy at different orders; something which is, in principle, not justified. 

by

Particularizing eq. (92), we have that the (2,0)-stiff effective free energy is given

$$
F_{\mathrm{s}}^{(2,0)}(s):=V_{\Sigma}(s)-T S_{\Sigma}^{\mathrm{k}}(s)-T S_{\mathcal{H}}^{\mathrm{c}}(s)
$$

with

$$
S_{\mathcal{H}}^{\mathrm{c}}(s):=-\frac{R}{2} \ln \operatorname{det} \mathcal{H}^{\Sigma}(s) .
$$

As we discuss in sec. 2.5, this model has been implicitly used in some works, including one by some us 48 .

The (2,1)-stiff free energy, in turn, includes an additional term:

$$
F_{\mathrm{s}}^{(2,1)}(s):=V_{\Sigma}(s)-T S_{\Sigma}^{\mathrm{k}}(s)-T S_{\mathcal{H}}^{\mathrm{c}}(s)+U_{\mathrm{s}}^{(1)}(s)
$$

defined as

$$
U_{\mathrm{s}}^{(1)}(s):=-\frac{1}{2 \beta^{2}} D\left(d^{I}(s)\right) \mathcal{H}_{\Sigma}^{I J}(s) D\left(d^{J}(s)\right),
$$

where we have used again that the first derivatives of the potential energy are zero in $\Sigma$, and $\mathcal{H}_{\Sigma}^{I J}(s)$ are the entries of the inverse matrix of the constrained Hessian, as usual. If the definition of the constrained subspace based in the minimization of $F(q)$ is used, then the $(2,0)$ - and (2,1)-stiff models become identical.

Now, if the modified coordinates $\tilde{q}$ defined in sec. 2.4.1 are used in the calculations in this section, several points need to be re-examined. First of all, we have the following relationship between the whole-space MMT in the coordinates $q$ and the one in the coordinates $\tilde{q}$ (indeed, we have it for any 2-times covariant tensor [46]):

$$
\tilde{G}_{\mu \nu}(\tilde{q})=\frac{\partial Q^{\rho}(\tilde{q})}{\partial \tilde{q}^{\mu}} G_{\rho \sigma}(Q(\tilde{q})) \frac{\partial Q^{\sigma}(\tilde{q})}{\partial \tilde{q}^{\nu}} .
$$

Hence, if we use the fact that the determinant of a product of square matrices is the product of the determinants, and also the property mentioned in sec. 2.4.1 about the Jacobian determinant of the change of coordinates being 1 , we have that

$$
\operatorname{det} \tilde{G}(\tilde{q})=\operatorname{det} G(Q(\tilde{q})) .
$$

Note that this not only guarantees the factorization of the external coordinates in eq. (31), but it also allows us to use the explicit expression (97) in terms of the molecular coordinates $q$ to compute $\operatorname{det} \tilde{G}(\tilde{q})$ as long as we insert the transformation functions $Q(\tilde{q})$ into the appropriate functional places corresponding to bond lengths and bond angles.

Also using the structure of the Jacobian in eq. $(75)$, it is easy to prove that the transformed potential energy,

$$
\tilde{V}(\tilde{q}):=V(Q(\tilde{q}))
$$

and indeed any scalar function (such as $\operatorname{det} G$ above, and hence $S^{\mathrm{k}}$ ), satisfies

$$
\frac{\partial \tilde{V}}{\partial \tilde{d}^{I}}(\tilde{q})=\frac{\partial V}{\partial d^{I}}(Q(\tilde{q})),
$$

as well as

$$
\frac{\partial^{2} \tilde{V}}{\partial \tilde{d}^{I} \partial \tilde{d}^{J}}(\tilde{q})=\frac{\partial^{2} V}{\partial d^{I} \partial d^{J}}(Q(\tilde{q})) .
$$

These transformation rules guarantee that all the final expressions in this section are identical in the case that we wished to use the modified coordinates $\tilde{q}$, as long 
as we 'place tildes' over all relevant objects, such as $\tilde{\mathcal{H}}(\tilde{q}), \tilde{A}(\tilde{q})$ or $\tilde{S^{\mathrm{k}}}(\tilde{q})$. But not only that, they also allow us to compute the objects in the original coordinates $q$ and then simply perform the substitution $Q(\tilde{q})$ to find the transformed quantities. This is very convenient, since the coordinates $\tilde{q}$ are defined in terms of the functions $f(s)$, which are typically impossible to express analytically, while the coordinates $q$ are simple functions of the Euclidean coordinates, thus admitting compact algorithms for computing quantities such as the Hessian of the potential energy, or the functions $D(d)$ and $D_{2}(d)$ in eqs. (101) and 103 , respectively.

It is also worth remarking that, in the derivation of the stiff models in the coordinates $\tilde{q}$, the Taylor expansion of the potential energy in eq. 86 would be performed at $\tilde{d}=0$ and take the simpler form

$$
\tilde{V}(\tilde{s}, \tilde{d})=\tilde{V}(\tilde{s}, 0)+\left[\frac{\partial \tilde{V}}{\partial \tilde{d}^{I}}(\tilde{s}, 0)\right] \tilde{d}^{I}+\left[\frac{\partial^{2} \tilde{V}}{\partial \tilde{d}^{I} \partial \tilde{d}^{J}}(\tilde{s}, 0)\right] \tilde{d}^{I} \tilde{d}^{J}+O\left(\tilde{d}^{3}\right)
$$

which allows to make contact with some of the potential energy functions in previous works 678889798369 , realizing that the intention of the authors may not be to restrict themselves to a particular case of the completely general potential considered here, but just that they are using the modified coordinates $\tilde{q}$, instead of the molecular coordinates $q$ used in this work. See however the discussion in sec. 2.5, about the neglection of the dependence of the Hessian on $\tilde{s}$, which is not a general feature of eq. (114), irrespective of the coordinates used.

Finally, to close this section, let us raise an important and subtle point related to the stiff model and the dynamics of the system: As we have emphasized in previous sections, the analysis presented in this work is completely at the equilibrium statistical mechanics level; all approximations are performed in equilibrium PDFs and all constrained models are defined in these terms too. In the previous rigid sections 2.4.1 and 2.4.2, we briefly commented that the rigid equilibrium can be sampled using available dynamical algorithms such as SHAKE 13063 140 or any of its more modern descendants (see ref. [78] and references therein) for the hard case; and the algorithms in refs. [504965] if the constraints are flexible. That this sampling can be achieved by such algorithms is easily understandable if we recall that the rigid model is obtained from a Hamiltonian [the one in eq. (49)] and thus the corresponding discretized Hamilton equations (properly thermostated [109]) will do the trick. Moreover, the fact that the fastest vibrations are absent from this dynamics makes the integration algorithms potentially more efficient than the unconstrained dynamics, and the whole scheme is thus useful.

In the case of the stiff model introduced in this section, the situation is entirely different. We may of course ask the theoretical question of which would be a dynamics that directly samples the stiff statistical mechanics equilibrium, and we may even find some dynamics that do the job. However, any naive version of such a dynamics will in principle contain the fast vibrations that we sought to eliminate with the use of constraints, and therefore any algorithm that implements it would probably be as numerically costly as the whole unconstrained dynamics (at least). Since the only rationale behind the development of the stiff model was to provide a good approximation to the unconstrained system in order to be able to assess the accuracy of the rigid equilibrium, using a new 'stiff' dynamics instead of the unconstrained one itself seems a rather unprofitable (and convoluted) enterprise. In principle, the stiff model should be regarded as an approximation to the unconstrained equilibrium at the level of statistical mechanics, not at the level of the dynamics. However, as we will discuss in sec. 2.5, there is a way of sampling the stiff equilibrium using a rigid dynamics with an additional correcting term (see fig. 3 for a schematic summary of these ideas). Whether or not the calculation of this correcting term can be performed 
fast enough so that we end with a dynamics which has the accuracy of the stiff model while retaining the efficiency of the rigid one is, however, a topic that we will not analyze in depth in this work.

\subsubsection{Stiff model with hard constraints}

In principle, we can also consider the stiff model defined using the hard constraints in eq. 80 . The main difference with the flexible case appears in the Taylor expansions in eq. (87) and (88), which become

$$
\begin{aligned}
V(s, d) & =\bar{V}_{\Sigma}(s)+\partial_{I} \bar{V}_{\Sigma}(s)\left(d^{I}-d_{0}^{I}\right) \\
& +\frac{1}{2} \overline{\mathcal{H}}_{I J}^{\Sigma}(s)\left(d^{I}-d_{0}^{I}\right)\left(d^{J}-d_{0}^{J}\right)+O\left(\left(d-d_{0}\right)^{3}\right),
\end{aligned}
$$

and

$$
\begin{aligned}
S^{\mathrm{k}}(s, d) & =\bar{S}_{\Sigma}^{\mathrm{k}}(s)+\partial_{I} \bar{S}_{\Sigma}^{\mathrm{k}}(s)\left(d^{I}-d_{0}^{I}\right) \\
& +\frac{1}{2} \partial_{I J}^{2} \bar{S}_{\Sigma}^{\mathrm{k}}(s)\left(d^{I}-d_{0}^{I}\right)\left(d^{J}-d_{0}^{J}\right)+O\left(\left(d-d_{0}\right)^{3}\right)
\end{aligned}
$$

where we have used again an over-bar to distinguish objects associated to the hard constraints from their flexible counterparts (meaning they are evaluated at $d=d_{0}$ instead of $d=f(s))$.

Also, since the values $d=d_{0}$ do not need to minimize the potential energy at the point $s$ [nor the free energy $F(q)]$, the derivatives $\partial_{I} \bar{V}_{\Sigma}(s), \partial_{I} \bar{S}_{\Sigma}^{\mathrm{k}}(s)$ and $\partial_{I} \bar{V}_{\Sigma}(s)-T \partial_{I} \bar{S}_{\Sigma}^{\mathrm{k}}(s)$ are all non-zero in general, and neither the Hessian $\overline{\mathcal{H}}^{\Sigma}(s)$ nor the associated matrix $\bar{A}(s)$ are guaranteed to be positive definite in the hard case.

All calculations for the hard stiff models are formally identical to the ones in the previous section as long as we include the terms $\partial_{I} \bar{V}_{\Sigma}(s)$ and $\partial_{I} \bar{S}_{\Sigma}^{\mathrm{k}}(s)$, and substitute the functions $f(s)$ by the constant values $d_{0}$.

In particular, the hard (2,2)-stiff model has free energy

$$
\bar{F}_{\mathrm{s}}^{(2,2)}(s):=\bar{V}_{\Sigma}(s)-T \bar{S}_{\Sigma}^{\mathrm{k}}(s)-T \bar{S}_{A}^{\mathrm{c}}(s)+\bar{U}_{\mathrm{s}}^{(2)}(s)
$$

with

$$
\begin{aligned}
\bar{S}_{\Sigma}^{\mathrm{k}}(s) & :=\frac{R}{2} \ln \operatorname{det} \bar{G}_{\Sigma}^{\prime}(s) \\
\bar{S}_{\mathrm{s}}^{\mathrm{c}}(s) & :=-\frac{R}{2} \ln \operatorname{det} \bar{A}(s)
\end{aligned}
$$

and

$$
\bar{U}_{\mathrm{s}}^{(2)}(s):=-\frac{1}{2}\left(\partial_{I} \bar{V}_{\Sigma}(s)-\frac{D\left(d_{0}^{I}\right)}{\beta}\right) \bar{A}^{I J}(s)\left(\partial_{J} \bar{V}_{\Sigma}(s)-\frac{D\left(d_{0}^{J}\right)}{\beta}\right)
$$

being

$$
\bar{A}_{I J}(s):=\overline{\mathcal{H}}_{I J}^{\Sigma}(s)+\frac{1}{\beta} \delta_{I J} D_{2}\left(d_{0}^{I}\right)
$$

As in the flexible case, the substitution of the second order expansion of the free energy into the integral in eq. 89 is only justified if $\bar{A}$ is positive definite and has large enough eigenvalues. In the case of the hard $(2,2)$-stiff model, the failure of such a property can be caused not only by the fact that the chosen constrained coordinates are actually not very 'stiff' or because we have decided to minimize $V(w)$ instead of 
$F(q)$ (as it happened for the flexible case in the previous section), but also because of having expanded around a value, $d_{0}$, which is not a minimum of the potential energy [or of $F(q)$ ], thereby possibly distorting the observed curvature encoded in $\overline{\mathcal{H}}^{\Sigma}$ and $\bar{A}$.

The only hard stiff model that presents an equilibrium PDF that is convergent for all points $s$ (but a partition function that is infinite) is the (0,0)-stiff model, whose free energy is given by

$$
\bar{F}_{\mathrm{s}}^{(0,0)}(s):=\bar{V}_{\Sigma}(s)-T \bar{S}_{\Sigma}^{\mathrm{k}}(s) .
$$

As in the flexible case, the first order truncation produces models that are, in general, non-convergent, while we can still define two models that may be well-behaved depending on the properties of the hard constrained Hessian $\overline{\mathcal{H}}^{\Sigma}$ : the $(2,0)$ - and $(2,1)$ stiff models. Notice, on the one hand, that these two models were convergent at all points $s$ in the flexible case, because the flexible constrained Hessian is, by construction, positive definite. On the other hand, the same caution applies in the hard case regarding the a priori justification of truncating the potential energy $V(s, d)$ and the kinetic entropy $S^{\mathrm{k}}(s, d)$ at different orders.

The hard (2,0)-stiff model has free energy

$$
\bar{F}_{\mathrm{s}}^{(2,0)}(s):=\bar{V}_{\Sigma}(s)-T \bar{S}_{\Sigma}^{\mathrm{k}}(s)-T \bar{S}_{\mathcal{H}}^{\mathrm{c}}(s)+\bar{U}_{\mathrm{s}}^{(0)}(s)
$$

with

$$
\bar{U}_{\mathrm{s}}^{(0)}(s):=-\frac{1}{2} \partial_{I} \bar{V}_{\Sigma}(s) \overline{\mathcal{H}}_{\Sigma}^{I J}(s) \partial_{J} \bar{V}_{\Sigma}(s)
$$

and

$$
\bar{S}_{\mathcal{H}}^{\mathrm{c}}(s):=-\frac{R}{2} \ln \operatorname{det} \overline{\mathcal{H}}^{\Sigma}(s)
$$

whereas the free energy of the hard (2,1)-stiff model is given by

$$
\bar{F}_{\mathrm{s}}^{(2,0)}(s):=\bar{V}_{\Sigma}(s)-T \bar{S}_{\Sigma}^{\mathrm{k}}(s)-T \bar{S}_{\mathcal{H}}^{\mathrm{c}}(s)+\bar{U}_{\mathrm{s}}^{(1)}(s)
$$

being

$$
\bar{U}_{\mathrm{s}}^{(1)}(s):=-\frac{1}{2}\left(\partial_{I} \bar{V}_{\Sigma}(s)-\frac{D\left(d_{0}^{I}\right)}{\beta}\right) \overline{\mathcal{H}}_{\Sigma}^{I J}(s)\left(\partial_{J} \bar{V}_{\Sigma}(s)-\frac{D\left(d_{0}^{J}\right)}{\beta}\right) .
$$

It is also worth noting here that, in the hard case treated in this section, there is no difference between minimizing $V(w)$ or $F(q)$, because the constrained subspace is not defined through any minimization, but it is postulated to simply be $d=d_{0}$.

Some of these models have been implicitly used in the literature before, as we mention in the next section. In fact, being the hard constraints more popular, their use have been more common than that of their flexible counterparts in sec. 2.4.3.

\subsection{Summary, comparisons and the Fixman's potential}

In fig. 3, a schematic depiction of the models discussed in the previous sections can be found, together with the relationships among them, as well as the basic mathematical expressions used to describe their respective statistical mechanics in the constrained subspace. In the figure, only the flexible versions of the constrained models have been detailed, and only the more accurate $(2,2)$-type of stiff model is shown. An equivalent scheme will be obtained if we define the constrained subspace $\Sigma$ using the hard relations $d=d_{0}$ or we truncate the Taylor expansions defining the stiff model at a different order than 2 . 


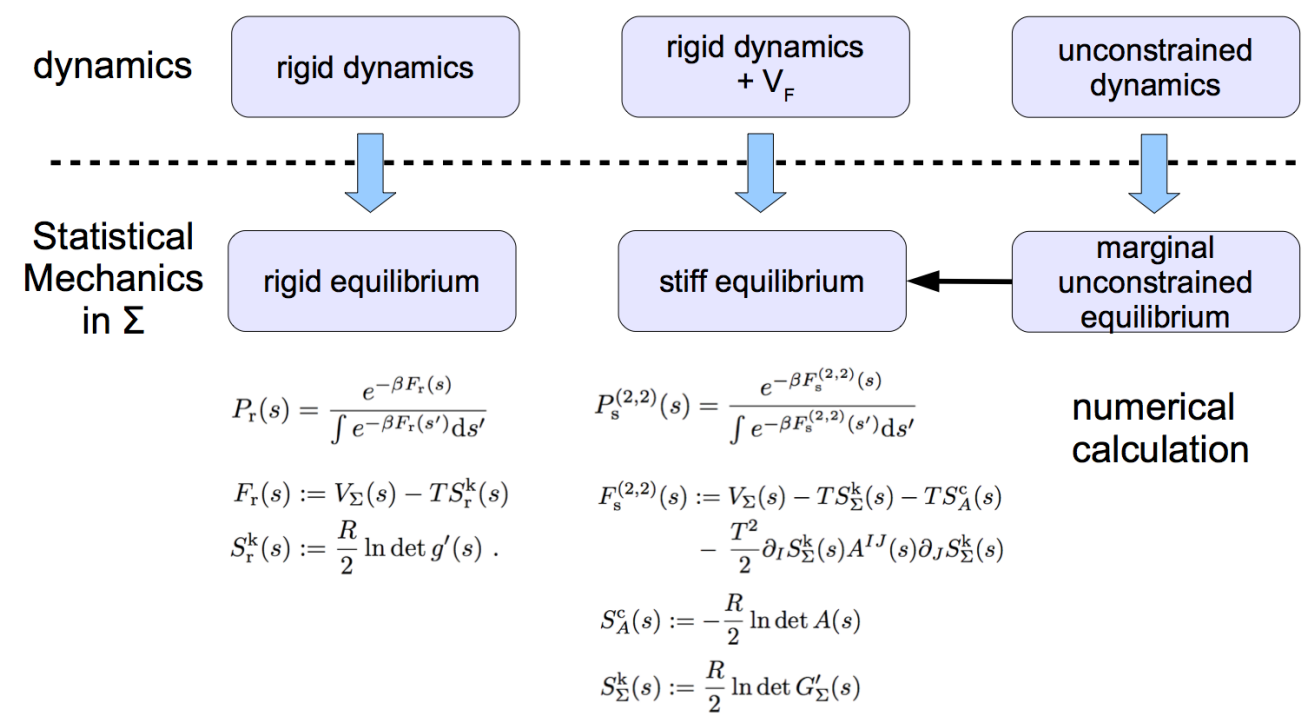

Fig. 3. Summary and relationships among the models discussed in this work. For a precise definition of the different mathematical objects and the meaning of the models, see the main body of the article.

In words, we have the unconstrained dynamics, which, we have argued, is the reference to which we will judge the accuracy of any constrained model. On the other end of the scheme, we have the rigid dynamics, which is potentially faster than the unconstrained one because the fastest vibrations have been eliminated from the system by constraining the associated degrees of freedom. Despite this better numerical profile, the rigid dynamics, and its corresponding statistical mechanics equilibrium in the constrained subspace $\Sigma$ are based on some questionable assumptions, such as D'Alembert's principle, or the imposition that the dynamics takes place exactly on $\Sigma$, thus forbidding the system to present velocities that are orthogonal to this space. These strong approximations require that we assess the accuracy of the rigid model against the reference unconstrained situation. In this work, we choose to do so at the level of equilibrium statistical mechanics, where the key object is the PDF and the free energy that appears in its exponent. Given the typical complexity of molecular potential energy functions, the integral needed to marginalize the unconstrained PDF to the constrained subspace $\Sigma$ and thus be able to compare it with the rigid one is too complicated to be performed analytically. This is why we have introduced an approximation to the unconstrained equilibrium, which has $\Sigma$ as its natural probability space, which is based in weaker assumptions than the rigid model, and which is termed stiff model. Now, by comparing the rigid equilibrium to the stiff one, the accuracy of the former can be assessed. That they are indeed appreciably different has long be known in the literature and many works have discussed it

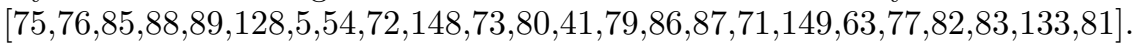

The most natural way to perform this assessment is to compare the two effective free energies that appear in the exponents of their respective PDFs. For example, if we consider the more general case of flexible constraints in secs. 2.4.1 and 2.4.3 and we use the most accurate $(2,2)$-stiff model, we can define the following function of the unconstrained coordinates $s$ :

$$
V_{\mathrm{F}}(s):=F_{\mathrm{s}}^{(2,2)}(s)-F_{\mathrm{r}}(s)=T S_{\mathrm{r}}^{\mathrm{k}}(s)-T S_{\Sigma}^{\mathrm{k}}(s)-T S_{A}^{\mathrm{c}}(s)+U_{\mathrm{s}}^{(2)}(s),
$$


which quantifies the difference between the two free energies, and contains many of the terms related to the MMT and Hessian determinants that we have introduced in the previous sections (but notice that the constrained potential energy $V_{\Sigma}(s)$ has been canceled out).

This function was first introduced by Fixman [128] (although in a much more simplified version) and it is thus normally called Fixman's potential. Apart from quantifying the discrepancies between the equilibrium statistical mechanics of the rigid model and the, in principle, more accurate stiff one, it can be easily seen that the addition of $V_{\mathrm{F}}(s)$ to the original potential energy $V_{\Sigma}(s)$ in the rigid dynamics produces an evolution of the system whose equilibrium is now given by the stiff PDF, at the same time that the fastest vibrations remain absent from the dynamics (assuming that $V_{\mathrm{F}}(s)$ has not reintroduced them). Indeed, if we modify the rigid Hamiltonian in eq. (49) and use the following one instead:

$$
H_{\mathrm{r}}^{\mathrm{F}}(u, \eta):=\frac{1}{2} \eta_{r} g^{r s}(u) \eta_{s}+V_{\Sigma}(s)+V_{\mathrm{F}}(s)
$$

it is immediate to see that the canonical equilibrium PDF of the corresponding dynamics (after integrating out the momenta and the external coordinates as we described in the previous sections) will be given by

$$
P_{\mathrm{r}}^{\mathrm{F}}(s)=\frac{e^{-\beta F_{\mathrm{r}}^{\mathrm{F}}(s)}}{\int e^{-\beta F_{\mathrm{r}}^{\mathrm{F}}\left(s^{\prime}\right)} \mathrm{d} s^{\prime}},
$$

where the obtained free energy $F_{\mathrm{r}}^{\mathrm{F}}(s)$ can be checked to be exactly the stiff one after some simple cancelations:

$$
\begin{aligned}
F_{\mathrm{r}}^{\mathrm{F}}(s) & =V_{\Sigma}(s)-T S_{\mathrm{r}}^{\mathrm{k}}(s)+V_{\mathrm{F}}(s) \\
& =V_{\Sigma}(s)-T S_{\mathrm{r}}^{\mathrm{k}}(s)+T S_{\mathrm{r}}^{\mathrm{k}}(s)-T S_{\Sigma}^{\mathrm{k}}(s)-T S_{A}^{\mathrm{c}}(s)+U_{\mathrm{s}}^{(2)}(s) \\
& =V_{\Sigma}(s)-T S_{\Sigma}^{\mathrm{k}}(s)-T S_{A}^{\mathrm{c}}(s)+U_{\mathrm{s}}^{(2)}(s) \\
& =: F_{\mathrm{s}}^{(2,2)}(s) .
\end{aligned}
$$

This practical use of the Fixman's potential to sample the stiff equilibrium using a modified rigid dynamics has often been discussed in the literature 13186149898877771857731057685804177669133 , and it is probable the main motivation behind its first introduction [128. In this work, however, no dynamical treatment is going to be performed, and we will use $V_{\mathrm{F}}(s)$ simply as a way of quantifying the difference between the stiff and rigid models at the level of equilibrium statistical mechanics. As we discussed before, this comparison can be understood as an assessment of the accuracy of the rigid equilibrium and, indirectly, as an evaluation of how much error the approximations behind the rigid model (including D'Alembert's principle) produce in the resulting statistical mechanics of the system.

We also point out that, if the justification of constrained models is not based in their comparison to the classical unconstrained case, as in this work, but to quantum mechanical calculations, then it can be argued that the equilibrium produced by the rigid dynamics is the 'correct' one, and therefore no corrections such as $V_{\mathrm{F}}(s)$ are needed [49. We discussed this issue in detail in sec. 2.3.2.

Apart from the introduction of $V_{\mathrm{F}}(s)$, Fixman proved in his celebrated paper 128 a theorem that, as we will see later, allows to simplify some MMT determinants in some cases. This useful result has been used in some works to compute certain PDFs even when no comparisons between stiff and rigid models are sought [1446331. Additionally, the issue of the comparison between constrained models in 
which the orthogonal velocities to the constrained subspace are activated and those in which they are assumed to be zero is central to the interpretation of the methods for computing free energy differences along a reaction coordinate 14431 150 32 33 146; however, despite the formal similarity, the reasons for imposing constraints and the physical considerations behind them are very different in the two cases. We suspect that these facts add even more confusion and complexity to some of the accounts about the topic.

It is also worth pointing out that the dynamics produced by the modified Hamiltonian in eq. (127) needs not to bear any resemblance to either the original rigid dynamics or the unconstrained one. The only convenient features of this dynamics, by construction, are the ones we have mentioned: (i) its equilibrium statistical mechanics is the stiff one, and (ii) assuming that $V_{\mathrm{F}}(s)$ does not introduce new fast oscillations, the dynamics is still rigid-like, in the sense that the fastest degrees of freedom have been removed and thus it can be integrated with a larger time-step than the unconstrained dynamics. In [40, for example, it is shown, under reasonable assumptions, that the averaged dynamics in the infinitely stiff case satisfies some modified equations of motion that evolve the trajectory in the constrained subspace, and whose modification is not the force corresponding to the Fixman's potential introduced here. This discrepancy could have several origins:

- The differences in the dynamics could be averaged out at equilibrium and the resulting PDF may turn out to be the same for the two cases [as we said, the dynamics produced by eq. (127) are only good for statistical mechanics sampling]. Since the dynamics in eq. (12) of [40] is, in principle, non-Hamiltonian, the techniques in [115] should be used to obtain its equilibrium PDF.

- As we mentioned in sec. 1 and we will develop further later in this section, the calculations in this work and the definition of Fixman's potential in eq. (126) are the most general in the literature as far as we are aware. In every other work, including [40, the version of the Fixman's potential that is used includes approximations. Therefore, a comparison of the modified dynamics in [40] to the one produced by the Hamiltonian in eq. (127) could yield different conclusions.

- Although the truncation of the Taylor expansion that took us to our definition of the stiff model in sec. 2.4 .3 seems very much related to the 'infinitely stiff' limit of the equations of motion in [40, there may exist subtle differences that could make the two physical situations sit on a different basis.

We do not pretend to solve this issue here, and the only reason why we have commented on it is to highlight the fact that dynamical analyses of constrained models are much more involved than the straightforward approach in this work, based in equilibrium properties. If one disregards the accuracy of the trajectories, the fact that the modified Hamiltonian in eq. (127) produces the stiff equilibrium through a constrained dynamics is very easy to prove and uncontroversial, as we have shown. Since the stiff model does not invoke in its derivation the D'Alembert principle, it is also clear that $V_{\mathrm{F}}(s)$ is an appropriate way of quantifying the accuracy of such an approximation; even if it were not the appropriate choice for doing the same thing at the dynamical level. It is also worth remarking that, doing similar dynamical analyses as those in 40, Reich finds a correcting term for the unconstrained coordinates which does seem to agree with the Fixman potential [14169.

Having these issues in mind, as we mentioned, the calculations in this work are the most general in the literature as far as we are aware. This is so because of a number of points:

- Apart from the requirement that they are 'stiff', no special property has been assumed about the constrained coordinates $d$. On the other hand, in many works 
6369678889128798283131 , the constraint functions $\sigma^{I}(w):=d^{I}-f^{I}(s)$ in eq. (71) have been chosen as the set $d$. In such a case, as we have discussed in the previous sections, many simplifications occur. For example, the induced MMT $g$ in the rigid models in secs. 2.4.1 and 2.4.2 is actually the unconstrainedunconstrained sub-block of the whole-space MMT restricted to $\mathcal{K}, G_{\mathcal{K}}$. Therefore, the theorem by Fixman [128] applies, and we have the following relation, which helps to more efficiently compute the difference $T S_{\mathrm{r}}^{\mathrm{k}}(s)-T S_{\Sigma}^{\mathrm{k}}(s)$ in $V_{\mathrm{F}}(s)$, and which has been used in many previous works [7576 6388899517986]:

$$
\frac{\operatorname{det} g^{\prime}(s)}{\operatorname{det} G_{\Sigma}^{\prime}(s)} \propto \frac{\operatorname{det} g(u)}{\operatorname{det} G_{\mathcal{K}}(u)}=\operatorname{det} h_{\mathcal{K}}(u),
$$

where $h$ denotes the constrained-constrained sub-block of $G^{-1}$ :

$$
h^{I J}:=\frac{\partial Q^{I}}{\partial r^{\mu}} \frac{1}{m_{\mu}} \frac{\partial Q^{J}}{\partial r^{\mu}} .
$$

The reason for expressing the above quotient of determinants as a function of det $h$ is that the entries of $h$ are in principle very easy to calculate if $q$ are the typical molecular coordinates consisting of bond lengths, bond angles, and dihedral angles [45], since each one of the functions $Q$ depends only on a few Euclidean coordinates $r$. This is however not true if the modified coordinates $\tilde{q}$ including the $\sigma$ functions are used. Indeed, if we compute the matrix $h$ in the $\tilde{q}$ coordinates and use the transformation rules in eq. 73 , we find that

$$
\begin{aligned}
\tilde{h}^{I J}(\tilde{q}):= & \frac{\partial \tilde{Q}^{I}}{\partial r^{\mu}}(R(\tilde{q})) \frac{1}{m_{\mu}} \frac{\partial \tilde{Q}^{J}}{\partial r^{\mu}}(R(\tilde{q})) \\
= & \frac{\partial \tilde{Q}^{I}}{\partial q^{\nu}}(Q(\tilde{q})) \frac{\partial Q^{\nu}}{\partial r^{\mu}}(R(\tilde{q})) \frac{1}{m_{\mu}} \frac{\partial Q^{\rho}}{\partial r^{\mu}}(R(\tilde{q})) \frac{\partial \tilde{Q}^{J}}{\partial q^{\rho}}(Q(\tilde{q})) \\
= & \frac{\partial f^{I}}{\partial \tilde{u}^{r}} \frac{\partial Q^{r}}{\partial r^{\mu}} \frac{1}{m_{\mu}} \frac{\partial Q^{s}}{\partial r^{\mu}} \frac{\partial f^{J}}{\partial \tilde{u}^{s}}-\frac{\partial Q^{I}}{\partial r^{\mu}} \frac{1}{m_{\mu}} \frac{\partial Q^{s}}{\partial r^{\mu}} \frac{\partial f^{J}}{\partial \tilde{u}^{s}} \\
& -\frac{\partial f^{I}}{\partial \tilde{u}^{r}} \frac{\partial Q^{r}}{\partial r^{\mu}} \frac{1}{m_{\mu}} \frac{\partial Q^{J}}{\partial r^{\mu}}+\frac{\partial Q^{I}}{\partial r^{\mu}} \frac{1}{m_{\mu}} \frac{\partial Q^{J}}{\partial r^{\mu}} \\
= & \frac{\partial f^{I}}{\partial \tilde{u}^{r}} h^{r s} \frac{\partial f^{J}}{\partial \tilde{u}^{s}}-h^{I s} \frac{\partial f^{J}}{\partial \tilde{u}^{s}}-\frac{\partial f^{I}}{\partial \tilde{u}^{r}} h^{r J}+h^{I J},
\end{aligned}
$$

where we have extended the definition in eq. (131) to run in the whole range $\mu, \nu=1, \ldots, N$, and we have dropped the dependencies towards the end of the calculation to make the expressions lighter. Now, we can conclude that, even if in the flexible case one can use the modified coordinates $\tilde{q}$ and the theorem by Fixman in eq. (130) still applies, it is not very useful. This is so because, although the terms related to the untransformed matrix $h$ in the above expression are still easy to compute, the derivatives $\partial f / \partial \tilde{u}$ are typically non-trivial, and they must be found numerically (see sec. 3 and ref. [116]). In the hard case, however, we have $f^{I}(s)=d_{0}^{I}$, and the relation between $\tilde{h}$ and $h$ becomes

$$
\tilde{h}^{I J}(\tilde{q})=h^{I J}(Q(\tilde{q})),
$$

I.e., in the hard case, eq. 130 is not only true, but also useful. In sec. II of ref. 73 , a very simple model illustrates how the choice of coordinates discussed in this point might have implications on the whole formalism apart from the Fixman theorem. In sec. 2.4 of ref. 67, the authors briefly agree with us about the fact that the use of the coordinates $\tilde{q}$ assumes that they have already been identified (computed), but this is non-trivial in general. 
- We have worked in the more general flexible setting, which includes the more restricted (and much more popular [123|124|75|125|78|61|126|62|127|76|85|58|63|128|5|72|80|59|90|129|86|87|71|77|130|13 hard models as a particular case. Note that, according to the discussion in sec.2.4.3. it is clear that this issue is coupled to the choice of coordinates discussed in the first point of this list. Indeed, if the modified coordinates $\tilde{q}$ introduced in the previous sections are used, we have that the Taylor expansion of the potential energy around the constrained subspace is given by eq. (114). Since the orders higher than 2 will also be multiplied by the undisplaced constrained coordinates $\tilde{d}$, we have that, in the coordinates $\tilde{q}$, the constraints defined through the minimization of the potential energy are effectively hard, i.e., $\tilde{d}=0$. However, despite this reformulation, the vast majority of the works in the literature do not refer to this, but actually to constraints with the form $d=d_{0}$ being the coordinates $d$ typically bond angles and bond lengths, which is not a reformulation but a restriction to a more particular case than the general, flexible one discussed here.

- The Taylor expansion used to build the stiff model in sec.2.4.3 has been truncated at the highest possible order compatible with the possibility of having analytical integrals, thus producing more accurate corrections, such as the ones including the derivatives of the determinant of the whole-space MMT $G$. This Taylor expansion of the term $-T S^{\mathrm{k}}(q)$ has not be accounted for in any previous work as far as we are aware (even in those works in which the treatment is most general [48/79|88|89|82[83]), thus effectively making the most accurate stiff model considered so far the $(2,0)$ one in secs. 2.4.3 and 2.4.4 Also, it is often assumed in the literature that the zero-order restriction to $\Sigma,-T S_{\Sigma}^{\mathrm{k}}(s)$, does not depend on $s$ 75/72 [90 129|87|133/134. The first approximation is wrong in general both in the flexible and hard cases [it is sufficient to look at eq. (97), where the dependence of $\operatorname{det} G^{\prime}$ on the typically constrained bond lengths and bond angles is explicit]. However, if hard constraints are chosen, and all bond lengths and bond angles are constrained, then $\operatorname{det} G^{\prime}$ is a constant and it can be canceled out from the expressions [75/72]129/87/71/77]. On the other hand, if at least some bond angles are considered unconstrained (as it is common in the literature [76/87/56/57/133]), we have that (i) $-T S^{\mathrm{k}}(q)$ depends on the constrained coordinates, thus suggesting the necessity of performing the Taylor expansion in eq. (88), and (ii) even the zero-order restriction to $\Sigma,-T S_{\Sigma}^{\mathrm{k}}(s)$, does depend on $s$, also in the hard case.

- The Taylor expansion mentioned in the previous point has also been performed here on the potential energy, thus producing correcting terms which include the Hessian matrix. In fact, since the best stiff model that appears in the previous literature is, as we mentioned, the $(2,0)$ one, the matrix $A$ in secs. 2.4 .3 and 2.4.4 is never found, only the Hessian (at most). This correction related to the Hessian has not been considered in a number of works, thus reducing the stiff model used to the (0,0)-type [75|85|63]128|5|80|90|131|133]134] (sometimes legitimately so, because of the restricted form of the potential energy used 71/86/87/77]), and it has been included (or at least mentioned) by some others [76/67/88/89|54|72]49|129|79|82|83|69. However, even in the second family of papers, it is often assumed that the Hessian does not depend on the unconstrained coordinates $s$ [76/72]129]69, which leads to effectively discard it. It is also worth pointing out that this last approximation is sometimes justified by the fact that the force field-like potential energy functions [such as the one in eq. [47]] typically have conformation-independent spring constants [76/72. However, even if we accept to particularize the calculations to classical force fields, the spring constants can only be assimilated to the Hessian in the infinite stiffness limit, which is not clear to be reached in force fields, specially for bond angles. Finally, we can think that the assumption that the zero-point energy of the quantum harmonic 
oscillators associated to the constrained coordinates is $s$-independent in quantum models is somehow related to this point [547249].

- The potential energy used to model the system and to define the constrained subspace in the flexible case has been considered to be completely general. It is common in the literature to explicitly or implicitly assume that a force field-like function [such as the one in eq. (47)] is used 69/76/67/88/89 128/54/72 41/79 83], or even simpler ones $718687 / 77$, which leads to more restricted versions of the objects computed here. For example, in ref. [71, (and similarly in [77) a polymer is considered whose potential energy is just the harmonic terms in eq. (47), i.e.,

$$
V(w):=\sum_{I} \frac{1}{2} K_{I}\left(d^{I}-d_{0}^{I}\right)^{2} .
$$

In such a case, the flexible constraints, as defined in this work, become $d=d_{0}$, i.e., exactly hard; with the associated simplifications discussed in the previous points. In the celebrated paper by Fixman [128, a similar choice is made. Also, as we mentioned in sec. 2.4.3, the choice of coordinates discussed in the first point of this list is related to the issue of the form potential energy. The Taylor expansion of the potential energy around the constrained subspace in the modified coordinates $\tilde{q}$ is given by eq. (114), which is in agreement with the potential used in some previous works 6788 8954417983, implying that maybe the authors were not considering a restriction to a force field-like function, but simply using the coordinates $\tilde{q}$. Remember however what we discussed about these coordinates in the first point, and also note that in some of these studies it is typically assumed that the Hessian is independent from the conformation [1169, which is not a general feature of eq. (114). In sec. II of ref. [73, the relationship between the coordinates chosen and the form of the potential energy is clearly illustrated using a simple 2-dimensional system, and see also sec.2.3.2 for more implications about this point in relation to flexible constraints.

In summary, in this work, all quantities appearing in the expressions: the MMT and Hessian determinants, the functions $f$, the derivatives of the potential energy, etc., have been considered to change with every variable that they can in principle depend on. No 'constancy' assumption has been made in our development. They have been expanded to the highest possible order compatible with analytical results, and no special form of the potential energy has been assumed.

The first issue in the above list affects the form of the final expressions and therefore complicates the 'translation' between our results and those in other works, but it does not introduce additional approximations. The rest of the assumptions (that we did not make here) effectively restrict the generality of the treatment, therefore potentially introducing modeling inaccuracies. Both types of issues typically produce simpler expressions and algorithms than the ones discussed here, being probably this practical benefit the original reason behind them. The most radical example of this is the case of a freely jointed chain with constrained bond lengths and bond angles, and no other potential energy than the harmonic oscillators in charge of enforcing these constraints [86 87/71/77/133134]. In such a situation, all terms in $V_{\mathrm{F}}(s)$ become independent from the conformation $s$ (the torsional angles in this case), except for $S_{\mathrm{r}}^{\mathrm{k}}(s)$; and therefore all the difference between the rigid and stiff models can be attached to $\operatorname{det} g$, which, in addition, can be very rapidly computed in terms of banded matrices 8687 .

Finally, it is worth remarking that, apart from the works that assume some of the approximations discussed above, the vast majority of constrained MD simulations in the literature do not consider any correcting term to $V_{\Sigma}$ at all; sometimes giving 
analytical or numerical reasons for it, sometimes simply not mentioning the issue [123 125|43/78|61|126|62|58|49|59|151|132|91|50].

\section{Numerical examples}

In this section, we provide a numerical example of the application of the general formalism introduced in this work to a real molecular system, using a classical force field.

It is worth mentioning at this point that many numerical studies about the equilibrium of constrained systems exist in the literature, each one of them containing some of the approximations which we carefully enumerated in the previous section, and which are avoided in this work.

Regarding the articles in which the stiff and rigid equilibrium distributions are compared, we can mention ref. [75], where a simplified model of $N$-butane is studied and the Fixman potential is found to be negligible; they use hard constraints, and they neglect both the Hessian and the $s$-dependence of $\operatorname{det} G$, effectively using the $(0,0)$-stiff model introduced in the previous sections. In ref. [85, the same molecule is studied, under the same approximating assumptions; they find that the probability of the trans-gauche transition state is changed in a $20-30 \%$ by the Fixman correction. $N$-butane is also analyzed in ref. [77, where they do not see an appreciable effect in transition rates or relaxation times if the Fixman potential is included; they assume again that the Hessian and the $s$-dependence of $\operatorname{det} G$ are negligible, and they impose hard constraints. Under the same assumptions, in ref. [133, the equilibrium of $N$ butane is shown to be altered by the inclusion of the Fixman correction if bond angles are constrained, but not if only bond lengths are (see below for more works discussing the bond angles issue). In ref. [73, the equilibrium distribution of certain angles in simplified models of three and four beads is found to be different between the stiff and rigid models. The same four-beads model is simulated in ref. [71, where the same comparison is performed and the rigid-plus-Fixman simulation is shown to be both equivalent to the unconstrained one, and different from the rigid simulation without correcting terms. In both ref. 73 and ref. [71, the simplicity of the model and the potential energy used to describe its behaviour make the hard and $(0,0)$ assumptions exact. In ref. [86, a linear freely jointed chain is considered, and the stiff torsional angles distribution is shown to be recovered from rigid simulations if the Fixman potential is included, which they find to have a non-negligible effect; they assume again the approximations that produce the $(0,0)$-stiff model, and they use hard constraints. The same system is studied in ref. [87, where Fixman's potential effects are measured as a function of the chain length, finding it non-negligible; again, the Hessian and the dependence of $\operatorname{det} G$ on $s$ are not considered.

A different but related family of works have numerically compared flexible and hard constraints. Among them, we can mention ref. 64, were they measure appreciable discrepancies between the two types of constraints in the calculation of the free energy difference between liquid water and methanol. In ref. [49], the unconstrained dynamics is taken as the reference to which the flexible and hard rigid models are compared in the computation of the velocity autocorrelation function of liquid water; the authors find that the predictions of the unconstrained and flexible rigid models are very similar, and different from those of the hard rigid case. In ref. [50] the same conclusion is drawn. In ref. 41, a simple four-beads system is studied and it is shown that the unconstrained average variation in bond angles is recovered if flexible constraints are used. A similar study in $N$-butane can be found in ref. 69, where the unconstrained distribution of the central torsion angle is shown to be well reproduced by the flexible rigid (plus Fixman) simulation, but not by the hard one. Finally, 


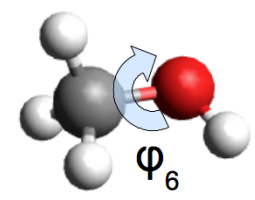

Fig. 4. The methanol molecule studied in this section. The only unconstrained coordinate, the dihedral angle $\varphi_{6}$, is indicated with a blue arrow.

in ref. [141, flexible constraints are proved to better reproduce the low-frequency part of the vibrational spectrum than hard ones in a simple toy model. The reader should notice that only in ref. 69 the Fixman potential is used to correct the rigid dynamics, which makes the agreement between the flexible rigid simulations and the unconstrained ones in the rest of works somehow surprising, probably suggesting that the Fixman correction is not significantly important in the particular systems that have been explored.

It is also worth to mention at this point the general agreement in the literature about bond angle constraints changing too much the equilibrium distribution with respect to the unconstrained case. Many works have studied this issue and have recommended not to constrain bond angle coordinates on the basis of their numerical findings [6/58 59:90|132 [91. However, all the simulations in these works have been performed (i) using hard constraints, and (ii) without including the Fixman correction. We have showed in this work that both these two assumptions should be considered approximations that might potentially compromise the accuracy of the final results. Therefore, we agree with the author of ref. 69. in suggesting the possibility that, perhaps, constraining bond angles can be made accurately if flexible constraints are used and the most general form of the Fixman correction introduced in this work is used to produce stiff averages (the numerical cost of such an approach is very relevant too, but it is independent from the accuracy issue). The checking of this hypothesis in biologically relevant molecules is currently in progress and it shall be reported elsewhere.

In the example calculation presented in this section, we have deliberately chosen a simple system in order to illustrate the theoretical concepts introduced in the previous sections. We study here the methanol molecule schematically depicted in fig. 4. and we compute its potential energy using the AMBER 96 force field [152/153]. The numeration of the atoms and the definition of the internal coordinates follow the SASMIC scheme, which is specially adapted to deal with constrained molecular systems [45. All the internal coordinates have been constrained, except for the principal dihedral angle, $\varphi_{6}$, which then becomes the only variable parameterizing the internal constrained subspace $\Sigma$. In order to produce the conformations in which all the quantities introduced in this work have been measured, we have scanned this dihedral angle from $0^{\circ}$ to $180^{\circ}$, in steps of $10^{\circ}$. At each point, we have generated the hard conformations simply by setting all the constrained coordinates to the constant values appearing in the force field, and the flexible conformations have been produced by minimizing the potential energy with respect to the constrained coordinates at fixed $\varphi_{6}$. These minimizations in internal coordinates, as well as the computation of the potential energy Hessian, have been performed with Gaussian 03 [154]. All correcting terms to the different statistical mechanics models discussed in the previous section have been computed using home-made programs.

First of all, we measured the most accurate version of the flexible stiff free energy in sec. 2.4.3. the quantity $F_{\mathrm{s}}^{(2,2)}(s)$, as a function of $\varphi_{6}$, as well as the Fixman potential, 


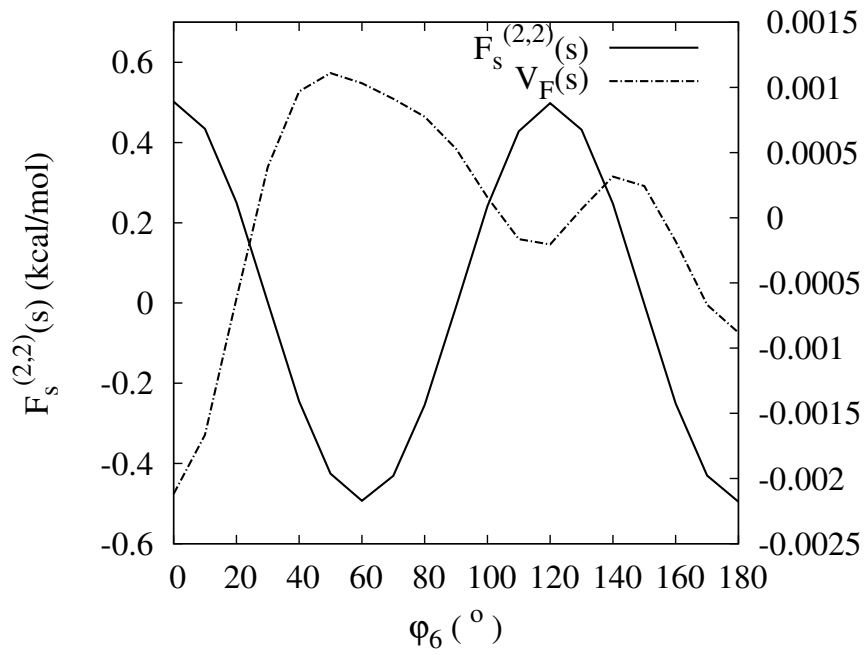

Fig. 5. Free energy, $F_{\mathrm{s}}^{(2,2)}(s)$, of the flexible $(2,2)$-stiff model, and Fixman's potential, $V_{\mathrm{F}}(s)$, as a function of the dihedral angle $\varphi_{6}$ in methanol. Both quantities have been added an irrelevant energy reference in order for them to have zero average.

\begin{tabular}{crrrr}
\hline & \multicolumn{2}{c}{ Flexible } & \multicolumn{2}{c}{ Hard } \\
& Std. $^{a}$ & Max. $^{b}$ & Std. $^{a}$ & Max. $^{b}$ \\
\hline$V_{\Sigma}$ & 0.37100819 & 1.02289469 & 0.37164847 & 1.02456429 \\
$-T S_{\mathrm{r}}^{\mathrm{k}}$ & 0.01116055 & 0.03295965 & 0.00000028 & 0.00000098 \\
$-T S_{\Sigma}^{\mathrm{k}}$ & 0.00008127 & 0.00022596 & 0.00000000 & 0.00000000 \\
$-T S_{\mathcal{H}}^{\mathrm{c}}$ & 0.01079596 & 0.03198016 & 0.01071757 & 0.03179323 \\
$-T S_{A}^{\mathrm{c}}$ & 0.01078666 & 0.03146866 & 0.01070879 & 0.03176306 \\
$U_{\mathrm{s}}^{(0)}$ & $\mathrm{N} / \mathrm{A}$ & $\mathrm{N} / \mathrm{A}$ & 0.00358154 & 0.01322298 \\
$U_{\mathrm{s}}^{(1)}$ & 0.00000004 & 0.00000013 & 0.00358563 & 0.01325454 \\
$U_{\mathrm{s}}^{(2)}$ & 0.00000004 & 0.00000013 & 0.00357814 & 0.01322534 \\
\hline
\end{tabular}

Table 2. ${ }^{a}$ Standard deviation, and ${ }^{b}$ maximum variation in the conformational space of the methanol molecule of the different terms introduced in sec. 2.4 which affect the equilibrium free energy of constrained models. All values are in $\mathrm{kcal} / \mathrm{mol}$.

$V_{\mathrm{F}}(s)$, in eq. (126), which quantifies the difference between the stiff equilibrium and the rigid one. The results, depicted in fig. 5. indicate that the variations in $V_{\mathrm{F}}(s)$ are at least two orders of magnitude smaller than those in $F_{\mathrm{s}}^{(2,2)}(s)$, and indeed much smaller than the thermal noise $R T \simeq 0.6 \mathrm{kcal} / \mathrm{mol}$ at room temperature, allowing us to conclude that, in this case, it is safe to neglect the Fixman correction. However, if we take a look at the variation of the different terms involved in the definition of $V_{\mathrm{F}}(s)$ in tab. 22 we see that $-T S_{\mathrm{r}}^{\mathrm{k}}(s)$ and $-T S_{A}^{\mathrm{c}}(s)$ show a variation which is one order of magnitude larger than that of $V_{\mathrm{F}}(s)$. What is happening is that these two quantities are much correlated in methanol and have opposite signs, thus almost canceling out. Preliminary data in larger systems indicates that this feature is only present in small molecules such as the one studied here. Also, the significance of $V_{\mathrm{F}}(s)$ appears to grow with molecular size. Therefore, the particular results in methanol should not be 

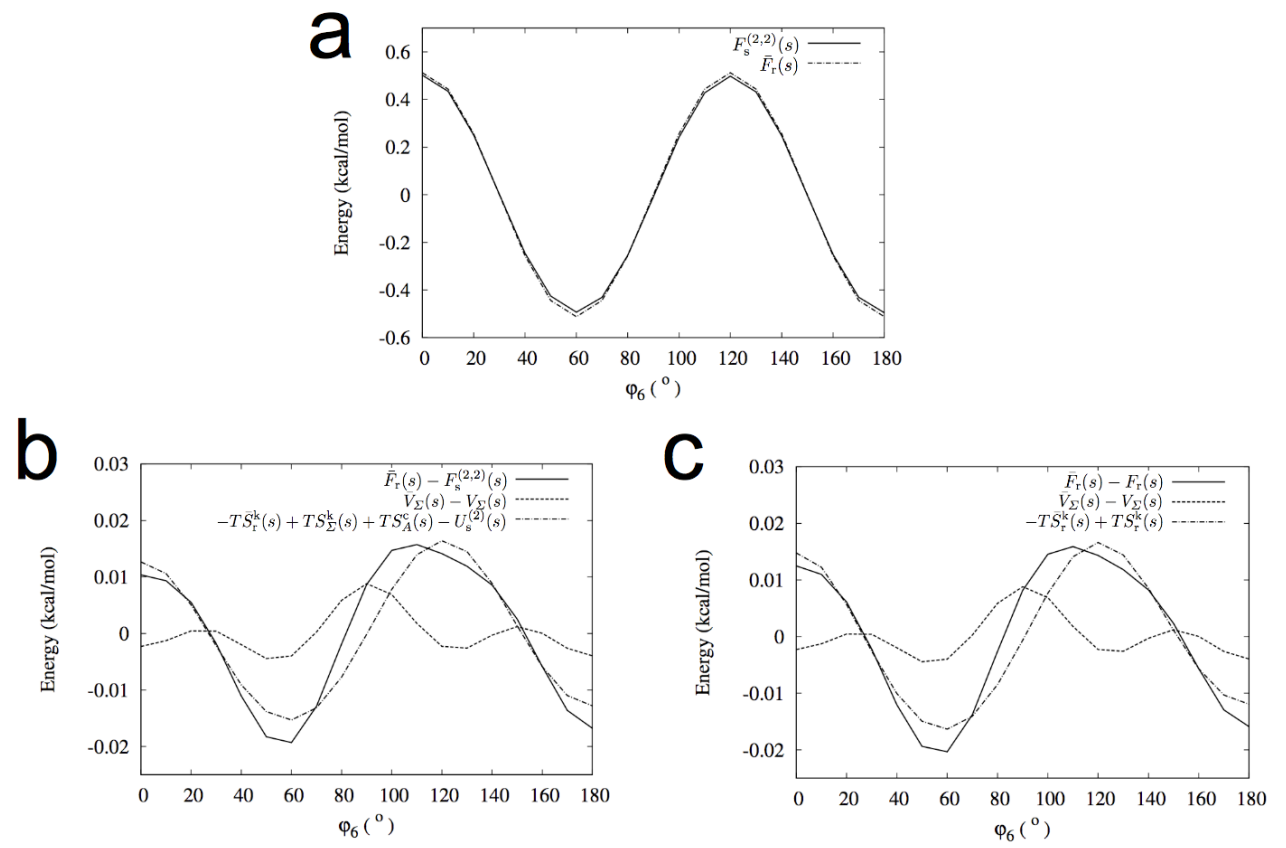

Fig. 6. Assessment of the hard rigid model for the methanol molecule. (a) Comparison between the flexible $(2,2)$-stiff free energy, $F_{\mathrm{s}}^{(2,2)}(s)$, and the hard rigid one, $\bar{F}_{\mathrm{r}}(s)$ as a function of the dihedral angle $\varphi_{6}$. (b) Difference between the two free energies in (a), as well as the difference between the potential energies, $\bar{V}_{\Sigma}(s)-V_{\Sigma}(s)$, and the correcting terms involved in the two models. (b) Difference between the flexible rigid free energy, $F_{\mathrm{r}}(s)$, and the hard rigid one, $\bar{F}_{\mathrm{r}}(s)$. Again the difference between the potential energies, $\bar{V}_{\Sigma}(s)-V_{\Sigma}(s)$, and the difference between the respective correcting terms are also depicted.

extrapolated in face value, but rather seen as an illustration of the concepts involved (probably the same can be said about the works discussed at the beginning of this section which analyze small toy systems and $N$-butane).

The difference between flexible and hard constraints in this molecule is larger than the difference between the stiff and rigid models, as we can appreciate in fig. 6, where the hard rigid model is compared both to the flexible $(2,2)$-stiff one (fig. $6 \mathrm{~b}$ ) and to the flexible rigid one (fig. 6r). In both cases, the maximum variation of the difference in the conformational space of methanol is approximately $0.04 \mathrm{kcal} / \mathrm{mol}$, an order of magnitude larger than the variation of $V_{\mathrm{F}}(s)$ in fig. 5. We can also see that both the difference between the flexible and hard potential energies, $\bar{V}_{\Sigma}(s)-V_{\Sigma}(s)$, and the difference between the correcting terms in the respective models contribute to the total discrepancy between the flexible and hard cases, being the second contribution somewhat larger than the first one. The fact that the graphs in figs. $6 \mathrm{p}$ and $6 \mathrm{c}$ seem identical to the eye (they are not exactly the same numerically) is again caused by the already mentioned correlation between the terms $-T S_{\mathrm{r}}^{\mathrm{k}}(s)$ and $-T S_{A}^{\mathrm{c}}(s)$ in methanol. Preliminary data in larger molecules suggest that these two comparisons are different in general, and also that the difference $\bar{V}_{\Sigma}(s)-V_{\Sigma}(s)$ becomes increasingly more important with system size if the bond angles remain among the constrained coordinates. This is probably due to the possibility of more steric clashes in longer, more flexible chains, and it is in agreement with the works discussing the bond angles issue that we have summarized at the beginning of this section. 

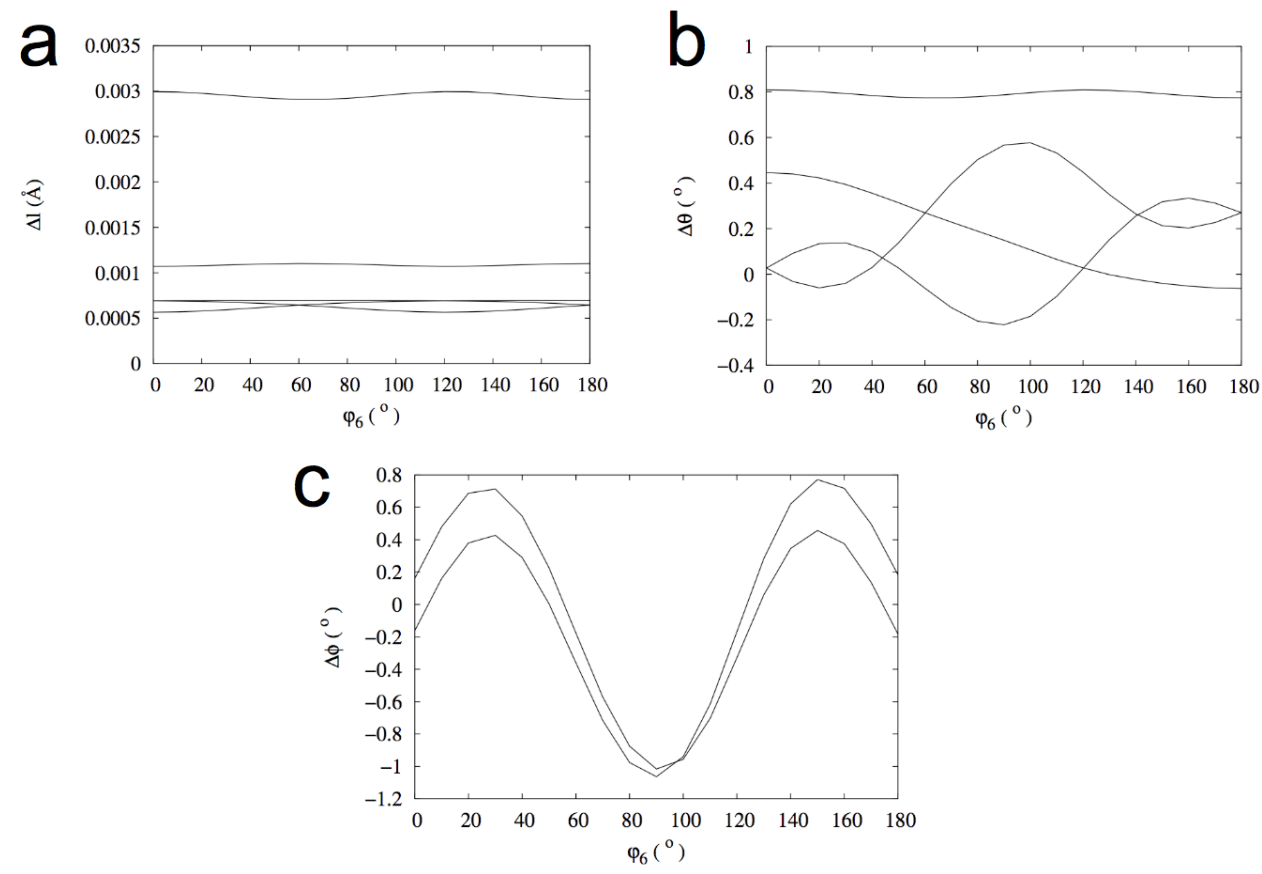

Fig. 7. Variation of the constrained coordinates in the flexible case in methanol, as a function of the unconstrained dihedral angle $\varphi_{6}$. (a) Variation of the bond lengths, (b) bond angles, and (c) phase dihedral angles with respect to the constant values appearing in the force field and defining the hard models.

Finally, we can see in fig. 7 that the constrained coordinates depend on the conformation in the more general flexible case, as expected from the discussion in sec. 2.3.2.

\section{Conclusions and future lines of research}

In this review, we have attempted to provide a unifying view of the statistical equilibrium of constrained classical mechanics models of molecular systems. To this end, we have introduced the most general formalism (i.e., including the fewest approximations) compatible with the possibility of having analytical results. From this advantageous standpoint, we have been able to rationalize most of the previous works in the literature, clearly identifying the underlying (often implicit) assumptions behind the different analyses. Also, we have tried to provide the reader with a coherent vocabulary which we hope facilitates future comparisons and reviews. Finally, we have shown a practical example of the different theoretical concepts.

Along the text, we have suggested a number of possible future lines of research that we believe could be interesting for distinct reasons, and some of which we have already started to pursue. We conclude this account by briefly collecting and commenting them:

- Define the constrained coordinates in an adaptive way, instead of simply picking whole sets of internal molecular coordinates of the same type (e.g., all bond angles involving hydrogens). If properly done, such an scheme could not only help to make calculations more accurate, but also to assess how good are the simpler approaches followed at the moment in the literature. 
- Develop a rigorous and general quantum mechanical derivation of constrained molecular models that could help decide which one of the classical models discussed in this work is more appropriately justified on physical grounds (if any of them).

- Compute the equilibrium PDF of the non-Hamiltonian, flexibly constrained dynamics described in ref. [49, maybe using the technique in ref. [115].

- Compare the option chosen in this work for defining the flexible constraints based on the minimization of the potential energy $V(w)$ to the alternative discussed in sec. 2.3.2, which uses the free energy $F(q)$ that appears in the exponent of the marginal PDF of the unconstrained dynamics in the space of the positions.

- Use techniques for the computation of free energy differences in order to numerically calculate the marginal PDF of the unconstrained dynamics in the space of the unconstrained coordinates $u$ [see eq. (85)], and compare it to the approximation to it provided by the stiff model.

- Solve the discrepancy between the correction to the rigid dynamics found from statistical analyses like the one in this work (i.e., the Fixman potential), and those obtained in some studies based on dynamical considerations, such as ref. [40. See also sec. 2.5 for further details.

- Extend the analysis in sec. 3 to larger, more relevant biological molecules.

- Explore the hypothesis, mentioned in sec. 3. that flexibly constraining bond angles and including the most general form of the Fixman potential to correct the rigid equilibrium may result in more accurate constrained simulations than the ones reported so far in the literature, where constraining bond angles is typically not recommended.

We thank J. L. Alonso, I. Calvo, G. Ciccotti, F. Falceto and B. Hess for illuminating discussions and advices regarding the mathematical formalism and the scope of the discussion presented here.

This work has been supported by the research projects E24/3 (DGA, Spain), FIS200913364-C02-01 (MICINN, Spain), 200980I064 (CSIC, Spain) and ARAID and Ibercaja grant for young researchers (Spain). P. G.-R. is supported by a JAE PREDOC grant (CSIC, Spain).

\section{References}

1. X. Andrade, A. Castro, D. Zueco, J.L. Alonso, P. Echenique, F. Falceto, A. Rubio, J. Chem. Theory Comput. 5, 728 (2009)

2. D.C. Rapaport, The art of molecular dynamics simulation, 2nd edn. (Cambridge University Press, 2004)

3. M.P. Allen, D.J. Tildesley, Computer simulation of liquids (Clarendon Press, Oxford, 2005)

4. T. Hundertmark, S. Reich, Eur. Phys. J. ST (2011), this issue.

5. D. Frenkel, B. Smit, Understanding molecular simulations: From algorithms to applications, 2nd edn. (Academic Press, Orlando FL, 2002)

6. P. Echenique, Contemp. Phys. 48, 81 (2007)

7. D.L. Ensign, P.M. Kasson, V.S. Pande, J. Mol. Biol. 374, 806 (2007)

8. P.L. Freddolino, K. Schulten, Biophys. J. 97, 2338 (2009)

9. D.E. Shaw, R.O. Dror, J.K. Salmon, J.P. Grossmann, K.M. Mackenzie, J.A. Bank, C. Young, M.M. Deneroff, B. Batson, K.J. Bowers et al., Millisecond-scale molecular dynamics simulations on Anton, in Proceedings of the ACM/IEEE Conference on Supercomputing (SC09) (Portland, Oregon, 2009), pp. 14-20

10. P. Echenique, J.L. Alonso, J. Comput. Chem. 29, 1408 (2008) 
11. J.L. Klepeis, K. Lindorff-Larsen, R.O. Dror, D.E. Shaw, Curr. Opin. Struct. Biol. 19, $1(2009)$

12. D. Marx, J. Hutter, Ab initio molecular dynamics: Theory and implementation, in Modern Methods and Algorithms of Quantum Chemistry, edited by J. Grotendorst (John von Neumann Institute for Computing, Jülich, 2000), Vol. 3, pp. 329-477

13. J.L. Alonso, X. Andrade, P. Echenique, F. Falceto, D. Prada-Gracia, A. Rubio, Phys. Rev. Lett. 101, 096403 (2008)

14. A.M.N. Niklasson, C.J. Tymczak, M. Challacombe, Phys. Rev. Lett. 97, 123001 (2006)

15. V.M. Anisimov, V.L. Bugaenko, C.N. Cavasotto, ChemPhysChem 10, 3194 (2009)

16. B.R. Brooks, C.L. Brooks III, A.D. MacKerell, L. Nilsson, R.J. Petrella, B. Roux, Y. Won, G. Archontis, C. Bartels, S. Boresch et al., J. Comput. Chem. 30, 1545 (2009)

17. W.L. Jorgensen, J. Tirado-Rives, J. Am. Chem. Soc. 110, 1657 (1988)

18. W.L. Jorgensen, D.S. Maxwell, J. Tirado-Rives, J. Am. Chem. Soc. 118, 11225 (1996)

19. J.W. Ponder, D.A. Case, Adv. Prot. Chem. 66, 27 (2003)

20. D.A. Case, T.A. Darden, T.E. Cheatham III, C.L. Simmerling, J. Wang, R.E. Duke, R. Luo, M. Crowley, R.C. Walker, W. Zhang et al., Amber 10, University of California, San Francisco (2008)

21. D.A. Pearlman, D.A. Case, J.W. Caldwell, W.R. Ross, T.E. Cheatham III, S. DeBolt, D. Ferguson, G. Seibel, P. Kollman, Comp. Phys. Commun. 91, 1 (1995)

22. F. Jensen, Introduction to Computational Chemistry (John Wiley \& Sons, Chichester, 1998)

23. P. Echenique, J.L. Alonso, Mol. Phys. 105, 3057 (2007)

24. A. Liwo, S. Oldziej, C. Czaplewski, D.S. Kleinerman, P. Blood, H.A. Scheraga, J. Chem. Theory Comput. p. Articles ASAP (2010)

25. C. Czaplewski, S. Kalinowski, A. Liwo, H.A. Scheraga, J. Chem. Theory Comput. 5, 627 (2009)

26. A. Emperador, O. Carrillo, M. Rueda, M. Orozco, Biophys. J. 95, 2127 (2008)

27. W. Han, C.K. Wan, Y.D. Wu, J. Chem. Theory Comput. 4, 1891 (2008)

28. J.A. de la Torre, G. Ciccotti, P. Español, M. Ferrario, Eur. Phys. J. ST (2011), this issue.

29. R. Car, M. Parrinello, Phys. Rev. Lett. 55, 2471 (1985)

30. J. Hutter, A. Curioni, ChemPhysChem 6, 1788 (2005)

31. E.A. Carter, G. Ciccotti, J.T. Hynes, R. Kapral, Chem. Phys. Lett. 5, 472 (1989)

32. C. Hartmann, G. Ciccotti, Eur. Phys. J. ST (2011), this issue.

33. J. Schlitter, Eur. Phys. J. ST (2011), this issue.

34. K.A. Dill, Prot. Sci. 8, 1166 (1999)

35. K.A. Dill, H.S. Chan, Nat. Struct. Biol. 4, 10 (1997)

36. C. Levinthal, How to fold graciously, in Mossbauer Spectroscopy in Biological Systems, edited by J.T.P. DeBrunner, E. Munck (University of Illinois Press, Allerton House, Monticello, Illinois, 1969), pp. 22-24

37. V. Daggett, A. Fersht, Nat. Rev. Mol. Cell Biol. 4, 497 (2003)

38. M. Karplus, J.A. McCammon, Nat. Struct. Biol. 9, 646 (2002)

39. T. Schlick, E. Barth, M. Mandziuk, Annu. Rev. Biophys. Biomol. Struct. 26, 181 (1997)

40. F.A. Bornemann, C. Schütte, Tech. rep., Konrad-Zuse-Zentrum für Informationstechnik Berlin (1995)

41. B.J. Leimkuhler, S. Reich, R.D. Skeel, in Mathematical approaches to biomolecular structure and dynamics, edited by J.P. Mesirov, K. Schulten (Springer, 1996)

42. M.M. Chawla, SIAM J. Numer. Anal. 22, 127 (1985)

43. K.A. Feenstra, B. Hess, H.J.C. Berendsen, J. Comput. Chem. 20, 786 (1999)

44. H. Goldstein, C. Poole, J. Safko, Classical Mechanics, 3rd edn. (Addison-Wesley, 2002)

45. P. Echenique, J.L. Alonso, J. Comput. Chem. 27, 1076 (2006)

46. B.A. Dubrovin, A.T. Fomenko, S.P. Novikov, Modern Geometry - Methods and Applications (Springer, Berlin, 1992)

47. E.W. Weisstein, Ordinary differential equations, from MathWorld - A Wolfram Web Resource. http://mathworld.wolfram.com/OrdinaryDifferentialEquations. html (last accessed on 03/17/09) 
48. P. Echenique, I. Calvo, J.L. Alonso, J. Comput. Chem. 27, 1748 (2006)

49. B. Hess, H. Saint-Martin, H.J.C. Berendsen, J. Chem. Phys. 116, 9602 (2002)

50. J. Zhou, S. Reich, B.R. Brooks, J. Chem. Phys. 112, 7919 (2000)

51. J.V. José, E.J. Saletan, Classical dynamics: a contemporary approach (Cambridge University Press, 1998)

52. R. Balescu, Equilibrium and nonequilibrium statistical mechanics (John Wiley \& Sons, New York, 1975)

53. K.B. Petersen, M.S. Pedersen, The matrix cookbook, Downoladed from http:// matrixcookbook.com (2010)

54. N. Gō, H.A. Scheraga, J. Chem. Phys. 51, 4751 (1969)

55. P. Echenique, I. Calvo, J. Comput. Chem. 27, 1733 (2006)

56. P. Eastman, V.S. Pande, J. Chem. Theory Comput. 6, 434 (2010)

57. B. Hess, J. Chem. Theory Comput. 4, 116 (2008)

58. J. Chen, W. Im, C.L. Brooks III, J. Comput. Chem. 26, 1565 (2005)

59. K. Hinsen, G.R. Kneller, Phys. Rev. E 52, 6868 (1995)

60. A.M. Mathiowetz, A. Jain, N. Karasawa, W.A. Goddard III, PROTEINS: Struct. Funct. Gen. 20, 227 (1994)

61. A.K. Mazur, R.A. Abagyan, J. Biomol. Struct. Dyn. 6, 815 (1989)

62. A.K. Mazur, J. Comput. Chem. 18, 1354 (1997)

63. G. Ciccotti, J.P. Ryckaert, Comput. Phys. Rep. 4, 345 (1986)

64. M. Christen, C.D. Christ, W.F. van Gunsteren, ChemPhysChem 8, 1557 (2007)

65. M. Christen, W.F. van Gunsteren, J. Chem. Phys. 122, 144106 (2005)

66. H. Saint-Martin, B. Hess, H.J.C. Berendsen, J. Chem. Phys. 120, 11133 (2004)

67. C.J. Cotter, S. Reich, BIT Num. Math. 44, 439 (2004)

68. U. Stocker, D. Juchli, W.F. van Gunsteren, Mol. Simul. 29, 123 (2003)

69. S. Reich, Phys. Rev. E 53, 53 (1996)

70. S. Reich, Num. Alg. 19, 213 (1998)

71. M.R. Pear, J.H. Weiner, J. Chem. Phys. 71, 212 (1979)

72. N. Gō, H.A. Scheraga, Macromolecules 9, 535 (1976)

73. E. Helfand, J. Chem. Phys. 71, 5000 (1979)

74. P. Pechukas, J. Chem. Phys. 72, 6320 (1980)

75. N.G. Almarza, E. Enciso, J. Alonso, F.J. Bermejo, M. Álvarez, Mol. Phys. 70, 485 (1990)

76. H.J.C. Berendsen, W.F. Van Gunsteren, in The Physics of Superionic Conductors and Electrode Materials, edited by J.W. Perram (Plenum Press, 1983), Vol. NATO ASI Series B92, pp. 221-240

77. D. Perchak, J. Skolnick, R. Yaris, Macromolecules 18, 519 (1985)

78. D. Dubbeldam, G.A.E. Oxford, R. Krishna, L.J. Broadbelt, R.Q. Snurr, J. Chem. Phys. 133, $034114(2010)$

79. D.C. Morse, Adv. Chem. Phys. 128, 65 (2004)

80. E.J. Hinch, J. Fluid Mech. 271, 219 (1994)

81. N.G. Van Kampen, J.J. Lodder, Am. J. Phys. 52, 419 (1984)

82. E.A.J.F. Peters, Ph.D. thesis, Technische Universiteit Delft (2000)

83. J.M. Rallison, J. Fluid Mech. 93, 251 (1979)

84. G. Gallavotti, The Elements of Mechanics (Ipparco Editore, 2007), available at http: //ipparco.roma1.infn.it/pagine/libri.html

85. D. Chandler, B.J. Berne, J. Chem. Phys. 71, 5386 (1979)

86. M. Pasquali, D.C. Morse, J. Chem. Phys. 116, 1834 (2002)

87. A. Patriciu, G.S. Chirikjian, R.V. Pappu, J. Chem. Phys. 121, 12708 (2004)

88. W.K. Den Otter, W.J. Briels, J. Chem. Phys. 109, 4139 (1998)

89. W.K. Den Otter, W.J. Briels, Mol. Phys. 98, 773 (2000)

90. M. Karplus, J.N. Kushick, Macromolecules 14, 325 (1981)

91. W.F. Van Gunsteren, M. Karplus, Macromolecules 15, 1528 (1982)

92. A.K. Mazur, B.G. Sumpter, D.W. Noid, Comput. Theor. Polym. Sci. 11, 35 (2001)

93. N. Saitô, K. Okano, S. Iwayanagi, T. Hideshima, Solid State Phys. 14, 343 (1963)

94. I.G. Tironi, R.M. Brunne, W.F. van Gunsteren, Chem. Phys. Lett. 250, 19 (1995) 
95. A.K. Mazur, in Computational Biochemistry and Biophysics, edited by O.M. Becker, A.D. MacKerell Jr., B. Roux, M. Watanabe (Marcel Dekker Inc., 2001)

96. R. Froese, I. Herbst, Commun. Math. Phys. 220, 489 (2001)

97. R.C.T. da Costa, Phys. Rev. A 25, 2893 (1982)

98. R.F. Álvarez-Estrada, Macromol. Theory Simul. 7, 457 (1998)

99. R.F. Álvarez-Estrada, Macromol. Theory Simul. 9, 83 (2000)

100. R.F. Álvarez-Estrada, G.F. Calvo, Mol. Phys. 100, 2957 (2002)

101. R.F. Álvarez-Estrada, G.F. Calvo, J. Phys.: Condens. Matter 16, S2037 (2004)

102. G.F. Calvo, R.F. Álvarez-Estrada, J. Phys.: Condens. Matter 17, 7755 (2005)

103. G.F. Calvo, R.F. Álvarez-Estrada, J. Phys.: Condens. Matter 20, 035101 (2008)

104. R.F. Álvarez-Estrada, G.F. Calvo, Eur. Phys. J. ST (2011), this issue.

105. M. Fixman, J. Chem. Phys. 69, 1527 (1978)

106. F.A. Bornemann, C. Schütte, Tech. rep., Konrad-Zuse-Zentrum für Informationstechnik Berlin (1995)

107. N.G. van Kampen, Phys. Rev. 124, 69 (1985)

108. R.D. Skeel, R. S., Eur. Phys. J. ST (2011), this issue.

109. J. Bajars, J. Frank, B. Leimkuhler, Eur. Phys. J. ST (2011), this issue.

110. J. Maddocks, C. Hartmann, Eur. Phys. J. ST (2011), this issue.

111. M.R. Flannery, Am. J. Phys. 73, 265 (2005)

112. W.S. Koon, J.E. Marsden, Rep. Math. Phys. 40, 21 (1997)

113. A.J. van der Schaft, B.M. Maschke, Rep. Math. Phys. 34, 225 (1994)

114. M. Molina-Becerra, E. Freire, J.G. Vioque, Equations of motion of nonholonomic Hamiltonian systems, Preprint obtained from http://www.matematicaaplicada2.es/ data/pdf/1276179170_1811485430.pdf (2010)

115. M.E. Tuckerman, Y. Liu, G. Ciccotti, G.J. Martyna, J. Chem. Phys. 115, 1678 (2001)

116. P. Echenique, C.N. Cavasotto, M. De Marco, P. García-Risueño, J.L. Alonso, An exact expression to calculate the derivatives of position-dependent observables in molecular simulations with flexible constraints, Submitted (2010)

117. T. Weise, Global optimization algorithms -theory and application-, Retrieved from http://www.it-weise.de (2009)

118. S. Kirkpatrick, C.D. Gelatt, M.P. Vecchi, Science 220, 671 (1983)

119. C.H. Yu, M.A. Norman, L. Schäfer, M. Ramek, A. Peeters, C. van Alsenoy, J. Mol. Struct. 567-568, $361(2001)$

120. L. Schäfer, M. Cao, M.J. Meadows, Biopolymers 35, 603 (1995)

121. L. Schäfer, M. Cao, M. Ramek, B.J. Teppen, S.Q. Newton, K. Siam, J. Mol. Struct. 413-414, 175 (1997)

122. A.T. Hagler, P.S. Stern, R. Sharon, J.M. Becker, F. Naider, J. Am. Chem. Soc. 101, $6842(1978)$

123. R.A. Abagyan, A.K. Mazur, J. Biomol. Struct. Dyn. 6, 833 (1989)

124. H. Abe, W. Braun, N. Gō, Comp. Chem. 8, 239 (1984)

125. R. Edberg, D.J. Evans, G.P. Morris, J. Chem. Phys. 84, 6933 (1986)

126. A.K. Mazur, V.E. Dorofeev, R.A. Abagyan, J. Comput. Phys. 92, 261 (1991)

127. T. Noguti, N. Gō, J. Phys. Soc. Japan 52, 3685 (1983)

128. M. Fixman, Proc. Natl. Acad. Sci. USA 71, 3050 (1974)

129. D.W. Li, R. Brüschweiler, Phys. Rev. Lett. 102, 118108 (2009)

130. J.P. Ryckaert, G. Ciccotti, H.J.C. Berendsen, J. Comput. Phys. 23, 327 (1977)

131. J. Schlitter, M. Klän, Mol. Phys. 101, 3439 (2003)

132. W.F. van Gunsteren, H.J.C. Berendsen, Mol. Phys. 34, 1311 (1977)

133. W.F. van Gunsteren, Mol. Phys. 40, 1015 (1980)

134. W.F. Van Gunsteren, in Computer Simulations of Biomolecular Systems, edited by W.F. Van Gunsteren, P.K. Weiner (Escom science publishers, Netherlands, 1989), pp. $27-59$

135. B. Hess, H. Saint-Martin, H.J.C. Berendsen, J. Chem. Phys. 120, 11133 (2004)

136. A. Szabo, N.S. Ostlund, Modern Quantum Chemistry: Introduced to Advanced Electronic Structure Theory (Dover Publications, New York, 1996) 
137. M. Ferrario, J.P. Ryckaert, Mol. Phys. 54, 587 (1985)

138. B. Hess, Private communication (2011)

139. T.Q. Yu, M. Tuckerman, Eur. Phys. J. ST (2011), this issue.

140. R. Elber, B. Hess, Eur. Phys. J. ST (2011), this issue.

141. S. Reich, Physica D 89, 28 (1995)

142. V.I. Arnold, Mathematical Methods of Classical Mechanics, Graduate Texts in Mathematics, 2nd edn. (Springer, New York, 1989)

143. H. Rubin, P. Ungar, Commun. Pure Appl. Math. X, 65 (1957)

144. G. Ciccotti, R. Kapral, E. Vanden-Eijnden, ChemPhysChem 6, 1809 (2005)

145. G.M. Torrie, J.P. Valleau, J. Comput. Phys. 23, 187 (1977)

146. J. Schlitter, M. Klän, J. Chem. Phys. 118, 2057 (2003)

147. I. Andricioaei, M. Karplus, J. Chem. Phys. 115, 6289 (2001)

148. O. Hassager, J. Chem. Phys. 60, 2111 (1974)

149. S. Reich, Physica D 118, 210 (2000)

150. C. Hartmann, C. Schütte, Physica D 228, 59 (2007)

151. S. Toxvaerd, O.J. Heilmann, T. Ingebrigtsen, T.B. Schrøder, J.C. Dyre, J. Chem. Phys. 131, 064102 (2009)

152. W.D. Cornell, P. Cieplak, C.I. Bayly, I.R. Gould, J. Merz, K. M., D.M. Ferguson, D.C. Spellmeyer, T. Fox, J.W. Caldwell, P.A. Kollman, J. Am. Chem. Soc. 117, 5179 (1995)

153. P. Kollman, R. Dixon, W. Cornell, T. Fox, C. Chipot, A. Pohorill, in Computer Simulations of Biomolecular Systems, edited by W.F. Van Gunsteren, P.K. Weiner, A.J. Wilkinson (Kluwer Academic Publishing, Dordrecht, 1997), Vol. 3, pp. 83-96

154. M.J. Frisch, G.W. Trucks, H.B. Schlegel, G.E. Scuseria, M.A. Robb, J.R. Cheeseman, J.A. Montgomery, Jr., T. Vreven, K.N. Kudin, J.C. Burant et al., Gaussian 03, Revision E.01 (2007), Gaussian, Inc., Wallingford, CT. 